J. Noncommut. Geom. 5 (2011), 69-105

DOI $10.4171 / \mathrm{JNCG} / 70$
Journal of Noncommutative Geometry

(C) European Mathematical Society

\title{
Van Den Bergh isomorphisms in string topology
}

\author{
Luc Menichi
}

\begin{abstract}
Let $M$ be a path-connected closed oriented $d$-dimensional smooth manifold and let $\mathbb{k}$ be a principal ideal domain. By Chas and Sullivan, the shifted free loop space homology of $M, H_{*+d}(\mathrm{~L} M)$ is a Batalin-Vilkovisky algebra. Let $G$ be a topological group such that $M$ is a classifying space of $G$. Denote by $S_{*}(G)$ the (normalized) singular chains on $G$. Suppose that $G$ is discrete or path-connected. We show that there is a Van Den Bergh type isomorphism
\end{abstract}

$$
\mathrm{HH}^{-p}\left(S_{*}(G), S_{*}(G)\right) \cong \mathrm{HH}_{p+d}\left(S_{*}(G), S_{*}(G)\right) .
$$

Therefore, the Gerstenhaber algebra $\mathrm{HH}^{*}\left(S_{*}(G), S_{*}(G)\right)$ is a Batalin-Vilkovisky algebra and we have a linear isomorphism

$$
\mathrm{HH}^{*}\left(S_{*}(G), S_{*}(G)\right) \cong H_{*+d}(\mathrm{~L} M) .
$$

This linear isomorphism is expected to be an isomorphism of Batalin-Vilkovisky algebras. We also give a new characterization of Batalin-Vilkovisky algebra in terms of the derived bracket.

Mathematics Subject Classification (2010). 55P50, 16E40, 16E45, 55P35, 57P10.

Keywords. String topology, Batalin-Vilkovisky algebra, Hochschild cohomology, free loop space, derived bracket, Van den Bergh duality, Poincaré duality group, Calabi-Yau algebra.

\section{Introduction}

We work over an arbitrary principal ideal domain $\mathbb{k}$. Let $M$ be a compact oriented $d$ dimensional smooth manifold. Denote by $L M:=\operatorname{map}\left(S^{1}, M\right)$ the free loop space on $M$. Chas and Sullivan [6] have shown that the shifted free loop homology $H_{*+d}(\mathrm{~L} M)$ has a structure of Batalin-Vilkovisky algebra (Definition 23). In particular, they showed that $H_{*+d}(\mathrm{~L} M)$ is a Gerstenhaber algebra (Definition 21). On the other hand, let $A$ be a differential graded (unital associative) algebra. The Hochschild cohomology of $A$ with coefficients in $A, \operatorname{HH}^{*}(A, A)$, is a Gerstenhaber algebra. These two Gerstenhaber algebras are expected to be related:

Conjecture 1. Let $G$ be a topological group such that $M$ is a classifying space of $G$. There is an isomorphism $H_{*+d}(\mathrm{~L} M) \cong \mathrm{HH}^{*}\left(S_{*}(G), S_{*}(G)\right)$ of Gerstenhaber algebras between the free loop space homology and the Hochschild cohomology of the algebra of singular chains on $G$. 
Suppose that $G$ is discrete or path-connected. In this paper, we define a BatalinVilkovisky algebra structure on $\mathrm{HH}^{*}\left(S_{*}(G), S_{*}(G)\right)$ and an isomorphism of graded k-modules

$$
\mathrm{BFG}^{-1} \circ \mathcal{D}: H_{*+d}(\mathrm{~L} M) \cong \mathrm{HH}^{*}\left(S_{*}(G), S_{*}(G)\right)
$$

which is compatible with the two $\Delta$ operators of the two Batalin-Vilkovisky algebras: $\mathrm{BFG}^{-1} \circ \mathscr{D} \circ \Delta=\Delta \circ \mathrm{BFG}^{-1} \circ \mathcal{D}$. Indeed, Burghelea, Fiedorowicz [5] and Goodwillie [19] gave an isomorphism of graded k-modules

$$
\mathrm{BFG}: \mathrm{HH}_{*}\left(S_{*}(G), S_{*}(G)\right) \stackrel{\cong}{\longrightarrow} H_{*}(\mathrm{~L} M)
$$

which interchanges Connes' boundary map $B$ and the $\Delta$ operator on $H_{*+d}(\mathrm{~L} M)$ : $\mathrm{BFG} \circ B=\Delta \circ \mathrm{BFG}$. And in this paper, our main result is:

Theorem 2 (Theorems 45 and 43). Let $G$ be a discrete or a path-connected topological group such that its classifying space BG is an oriented Poincaré duality space of formal dimension $d$. Then the following holds:

a) There exist $\mathbb{k}$-linear isomorphisms

$$
\mathcal{D}: \mathrm{HH}_{d-p}\left(S_{*}(G), S_{*}(G)\right) \stackrel{\cong}{\longrightarrow} \mathrm{HH}^{p}\left(S_{*}(G), S_{*}(G)\right) .
$$

b) Let $B$ denote the Connes boundary map on $\mathrm{HH}_{*}\left(S_{*}(G), S_{*}(G)\right)$. Then $\Delta:=-\mathscr{D} \circ B \circ \mathscr{D}^{-1}$ defines the structure of a Batalin-Vilkovisky algebra on $\mathrm{HH}^{*}\left(S_{*}(G), S_{*}(G)\right)$, extending the canonical Gerstenhaber algebra structure.

c) The cyclic homology $\mathrm{HC}_{*}\left(S_{*}(G)\right)$ of $S_{*}(G)$ has a Lie bracket of degree $2-d$.

By [33], Proposition 28, c) follows directly from b). Note that when $G$ is a discrete group, the algebra $S_{*}(G)$ of normalized singular chains on $G$ is just the group ring $\mathrm{k}[G]$.

To prove Conjecture 1 in the discrete or path-connected case, it suffices now to show that the composite $\mathrm{BFG}^{-1} \circ \mathscr{D}$ is a morphism of graded algebras. When $\mathbb{k}$ is a field of characteristic 0 and $G$ is discrete, this was proved by Vaintrob [39].

Suppose now that

$$
M \text { is simply-connected and that } \mathbb{k} \text { is a field. }
$$

In this case, there is a more famous dual conjecture relating Hochschild cohomology and string topology.

Conjecture 4. Suppose that (3) holds. Then there is an isomorphism $H_{*+d}(\mathrm{~L} M) \cong$ $\mathrm{HH}^{*}\left(S^{*}(M), S^{*}(M)\right)$ of Gerstenhaber algebras between the free loop space homology and the Hochschild cohomology of the algebra of singular cochains on $M$.

In fact, Theorem 2 is the Eckmann-Hilton or Koszul dual of the following theorem. 
Theorem 5 ([13], Theorem 23, and [33], Theorem 22). Assume (3).

a) There exist isomorphism of graded $\mathbb{k}$-vector spaces

FTV: $\operatorname{HH}^{p-d}\left(S^{*}(M), S^{*}(M)^{\vee}\right) \stackrel{\cong}{\longrightarrow} \mathrm{HH}^{p}\left(S^{*}(M), S^{*}(M)\right)$.

b) The Connes coboundary $B^{\vee}$ on $\mathrm{HH}^{*}\left(S^{*}(M), S^{*}(M)^{\vee}\right)$ defines via the isomorphism FTV a structure of Batalin-Vilkovisky algebra extending the Gerstenhaber algebra $\mathrm{HH}^{*}\left(S^{*}(M), S^{*}(M)\right)$.

Jones [23] proved that there is an isomorphism

$$
J: H_{p+d}(\mathrm{~L} M) \stackrel{\cong}{\longrightarrow} \mathrm{HH}^{-p-d}\left(S^{*}(M), S^{*}(M)^{\vee}\right)
$$

such that the $\Delta$ operator of the Batalin-Vilkovisky algebra $H_{*+d}(\mathrm{~L} M)$ and Connes' coboundary map $B^{\vee}$ on $\mathrm{HH}^{*-d}\left(S^{*}(M), S^{*}(M)^{\vee}\right)$ satisfy $J \circ \Delta=B^{\vee} \circ J$. Therefore, as we explain in [33], to prove Conjecture 4, it suffices to show that the composite FTV $\circ J$ is a morphism of graded algebras.

In [12], together with Felix and Thomas, we prove that Hochschild cohomology satisfies some Eckmann-Hilton or Koszul duality.

Theorem 6 ([12], Corollary 2; see also [7], Theorem 69, and below). Let $\mathbb{k}$ be a field. Let $G$ be a connected topological group. Denote by $S^{*}(B G)$ the algebra of singular cochains on the classifying space of $G$. Suppose that $H_{i}(B G)$ is finite dimensional for all $i \in \mathbb{N}$. Then there exists an isomorphism of Gerstenhaber algebras

$$
\text { Gerst: } \mathrm{HH}^{*}\left(S_{*}(G), S_{*}(G)\right) \stackrel{\cong}{\Longrightarrow} \mathrm{HH}^{*}\left(S^{*}(B G), S^{*}(B G)\right) \text {. }
$$

Therefore under (3), Conjectures 4 and 1 are equivalent, and, under (3), Theorem 2 as stated in this introduction follows from Theorem 5 .

The problem is that the isomorphism Gerst in Theorem 6 does not admit a simple formula. On the contrary, as we explain in Theorems 45 and 43, in this paper the isomorphism $\mathscr{D}$ is very simple: $\mathscr{D}^{-1}$ is given by the cap product with a fundamental class $c \in \mathrm{HH}_{d}\left(S_{*}(G), S_{*}(G)\right)$.

In [18], Theorem 3.4.3 (i), Ginzburg (see also [26], Proposition 1.4) shows that the Van den Bergh duality isomorphism $\mathscr{D}: \mathrm{HH}_{d-p}(A, A) \stackrel{\cong}{\Longrightarrow} \operatorname{HH}^{p}(A, A)$ is $\mathrm{HH}^{*}(A, A)$-linear for any Calabi-Yau algebra $A: \mathscr{D}^{-1}$ is also given by the cap product with a fundamental class $c \in \mathrm{HH}_{d}(A, A)$.

We now give the plan of the paper.

In Section 2 we recall the definitions of the bar construction, of the Hochschild (co)chain complex and of Hochschild (co)homology.

In Section 3 we show that, for some augmented differential graded algebra $A$ such that the dual of its reduced bar construction $B(A)^{\vee}$ satisfies the Poincare duality, we have a Van den Bergh duality isomorphism $\operatorname{HH}_{d-p}(A, A) \cong \operatorname{HH}^{p}(A, A)$ if $A$ is connected (Corollaries 13 and 14). 
There is a well-known isomorphism between group (co)homology and Hochschild (co)homology. In Section 4 we show that, through this isomorphism, cap products in Hochschild (co)homology correspond to cap products in group (co)homology.

In Section 5 we give a new characterization of Batalin-Vilkovisky algebras.

Ginzburg proved that if Hochschild (co)homology satisfies a Van den Bergh duality isomorphism $\mathrm{HH}_{d-p}(A, A) \cong \mathrm{HH}^{p}(A, A)$ then Hochschild cohomology has a Batalin-Vilkovisky algebra structure. In Section 6 we rewrite the proof of Ginzburg using our new characterization of Batalin-Vilkovisky algebras and insisting on signs.

In Section 7 we show that a differential graded algebra quasi-isomorphic to an algebra satisfying Poincaré duality, also satisfies Poincaré duality (Proposition 41). Finally, we show our main theorem for path-connected topological group.

In Section 8 we show our main theorem for discrete groups. Extending a result of Kontsevich [18], Corollary 6.1.4, and Lambre [26], Lemme 6.2, we also show that, over any commutative ring $\mathbb{k}$, the group ring $\mathbb{k}[G]$ of an orientable Poincaré duality group is a Calabi-Yau algebra.

Let $G$ be a path-connected compact Lie group of dimension $d$. In Section 9 we give another Van Den Bergh type isomorphism

$$
\mathrm{HH}^{p}\left(S^{*}(B G), S^{*}(B G)\right) \cong \mathrm{HH}_{-d-p}\left(S^{*}(B G), S^{*}(B G)\right) .
$$

Therefore, the Gerstenhaber algebra $\operatorname{HH}^{*}\left(S^{*}(B G), S^{*}(B G)\right)$ is a Batalin-Vilkovisky algebra and we have a linear isomorphism

$$
\mathrm{HH}^{*}\left(S^{*}(B G), S^{*}(B G)\right) \cong H^{*+d}(\mathrm{LBG}) .
$$

In the Appendix, Section 10, we recall the notion of derived bracket following Kosmann-Schwarzbach [24]. We interpret our new characterization of BatalinVilkovisky algebra in terms of the derived bracket (Theorem 66). To any differential graded algebra $A$, we associate

- a new Lie bracket on $A$ (Remark 63),

- a new Gerstenhaber algebra which is a subalgebra of the endomorphism algebra of $\mathrm{HH}_{*}(A, A)$ (Theorem 67).

We conjecture that Theorem 2 is true without assuming that $G$ is discrete or pathconnected. Note that the proof of the discrete case (Sections 4 and 8) is independent of the proof of the path-connected case (Sections 3 and 7).

Acknowledgment. We wish to thank Jean-Claude Thomas for several discussions, in particular for pointing out the Mittag-Leffler condition which is the key to Proposition 12.

\section{Hochschild homology and cohomology}

We work over an arbitrary commutative ring $\mathbb{k}$ except in Sections 3 and 7 , where $\mathbb{k}$ is assumed to be a principal ideal domain and in Section 9 where $k$ is assumed to 
be a field. We use the graded differential algebra of [11], Chapter 3. In particular, an element of lower degree $i \in \mathbb{Z}$ is by the classical convention [11], p. 41-42, of upper degree $-i$. Differentials are of lower degree -1 . All the algebras considered in this paper, are unital and associative. Let $A$ be a differential graded algebra. Let $M$ be a right $A$-module and $N$ be a left $A$-module. Denote by s $A$ the suspension of $A,(\mathrm{~s} A)_{i}=A_{i-1}$. Let $d_{0}$ be the differential on the tensor product of complexes $M \otimes T(\mathrm{~s} A) \otimes N$. We denote the tensor product of the elements $m \in M, s a_{1} \in \mathrm{s} A$, $\ldots, s a_{k} \in \mathrm{s} A$ and $n \in N$ by $m\left[a_{1}|\ldots| a_{k}\right] n$. Let $d_{1}$ be the differential on the graded vector space $M \otimes T(\mathrm{~s} A) \otimes N$ defined by

$$
\begin{aligned}
d_{1} m\left[a_{1}|\ldots| a_{k}\right] n= & (-1)^{|m|} m a_{1}\left[a_{2}|\ldots| a_{k}\right] n \\
& +\sum_{i=1}^{k-1}(-1)^{\varepsilon_{i}} m\left[a_{1}|\ldots| a_{i} a_{i+1}|\ldots| a_{k}\right] n \\
& -(-1)^{\varepsilon_{k-1}} m\left[a_{1}|\ldots| a_{k-1}\right] a_{k} n,
\end{aligned}
$$

where $\varepsilon_{i}=|m|+\left|a_{1}\right|+\cdots+\left|a_{i}\right|+i$.

The bar construction of $A$ with coefficients in $M$ and in $N$, denoted $B(M ; A ; N)$, is the complex $\left(M \otimes T(\mathrm{~s} A) \otimes N, d_{0}+d_{1}\right)$. The bar resolution of $A$, denoted $B(A ; A ; A)$, is the differential graded $(A, A)$-bimodule $\left(A \otimes T(\mathrm{~s} A) \otimes A, d_{0}+d_{1}\right)$. If $A$ is augmented then the reduced bar construction of $A$, denoted $B(A)$, is $B(\mathbb{k} ; A ; \mathbb{k})$.

Denote by $A^{\text {op }}$ the opposite algebra of $A$ and by $A^{e}:=A \otimes A^{\text {op }}$ the enveloping algebra of $A$. Let $M$ be a differential graded $(A, A)$-bimodule. Recall that any ( $A, A$ )-bimodule can be considered as a left (or right) $A^{e}$-module. The Hochschild chain complex is the complex $M \otimes_{A^{e}} B(A ; A ; A)$ denoted $\mathcal{C}_{*}(A, M)$. Explicitly $\mathcal{C}_{*}(A, M)$ is the complex $\left(M \otimes T(\mathrm{~s} A), d_{0}+d_{1}\right)$ with $d_{0}$ obtained by tensorization and [8], (10), p. 78,

$$
\begin{aligned}
d_{1} m\left[a_{1}|\ldots| a_{k}\right]= & (-1)^{|m|} m a_{1}\left[a_{2}|\ldots| a_{k}\right]+\sum_{i=1}^{k-1}(-1)^{\varepsilon_{i}} m\left[a_{1}|\ldots| a_{i} a_{i+1}|\ldots| a_{k}\right] \\
& -(-1)^{\left|s a_{k}\right| \varepsilon_{k-1}} a_{k} m\left[a_{1}|\ldots| a_{k-1}\right] .
\end{aligned}
$$

The Hochschild homology of $A$ with coefficients in $M$ is the homology $H$ of the Hochschild chain complex:

$$
\mathrm{HH}_{*}(A, M):=H\left(\mathcal{C}_{*}(A, M)\right) .
$$

The Hochschild cochain complex of $A$ with coefficients in $M$, denoted by $\ell^{*}(A, M)$, is the complex $\operatorname{Hom}_{A^{e}}(B(A ; A ; A), M)$. Explicitly $\ell^{*}(A, M)$ is the complex

$$
\left(\operatorname{Hom}(T(\mathrm{~s} A), M), D_{0}+D_{1}\right) .
$$

Here for $f \in \operatorname{Hom}(T(\mathrm{~s} A), M), D_{0}(f)([])=d_{M}(f([])), D_{1}(f)([])=0$, and for 
$k \geq 1$ we have

$$
\begin{aligned}
D_{0}(f)\left(\left[a_{1}\left|a_{2}\right| \ldots \mid a_{k}\right]\right)= & d_{M}\left(f\left(\left[a_{1}\left|a_{2}\right| \ldots \mid a_{k}\right]\right)\right) \\
& -\sum_{i=1}^{k}(-1)^{\bar{\epsilon}_{i}} f\left(\left[a_{1}|\ldots| d_{A} a_{i}|\ldots| a_{k}\right]\right)
\end{aligned}
$$

and

$$
\begin{aligned}
D_{1}(f)\left(\left[a_{1}\left|a_{2}\right| \ldots \mid a_{k}\right]\right)= & -(-1)^{\left|s a_{1}\right||f|} a_{1} f\left(\left[a_{2}|\ldots| a_{k}\right]\right) \\
& -\sum_{i=2}^{k}(-1)^{\bar{\epsilon}_{i}} f\left(\left[a_{1}|\ldots| a_{i-1} a_{i}|\ldots| a_{k}\right]\right) \\
& +(-1)^{\bar{\epsilon}_{k}} f\left(\left[a_{1}\left|a_{2}\right| \ldots \mid a_{k-1}\right]\right) a_{k},
\end{aligned}
$$

where $\bar{\epsilon}_{i}=|f|+\left|s a_{1}\right|+\left|s a_{2}\right|+\cdots+\left|s a_{i-1}\right|$.

The Hochschild cohomology of $A$ with coefficients in $M$ is

$$
\mathrm{HH}^{*}(A, M)=H\left(\mathcal{C}^{*}(A, M)\right) .
$$

Suppose that $A$ has an augmentation $\varepsilon: A \rightarrow \mathbb{k}$. Let $\bar{A}:=\operatorname{ker} \varepsilon$ be the augmentation ideal. We denote by $\bar{B}(A):=\left(T s \bar{A}, d_{0}+d_{1}\right)$ the normalized reduced bar construction, by $\bar{\ell}_{*}(A, M):=\left(M \otimes T(s \bar{A}), d_{0}+d_{1}\right)$ the normalized Hochschild chain complex and by $\bar{\complement}^{*}(A, M):=\left(\operatorname{Hom}(T(s \bar{A}), M), D_{0}+D_{1}\right)$ the normalized Hochschild cochain complex.

\section{The isomorphism between Hochschild cohomology and Hochschild homology for differential graded algebras}

Let $A$ be a differential graded algebra. Let $P$ and $Q$ be two $A$-bimodules.

The action of $\mathrm{HH}^{*}(A, Q)$ on $\mathrm{HH}_{*}(A, P)$ comes from a (right) action of the $\ell^{*}(A, Q)$ on $\ell_{*}(A, P)$ given by [8], (18), p. 82, [26],

$$
\begin{aligned}
\cap: \mathcal{C}_{*}(A, P) \otimes \mathcal{C}^{*}(A, Q) & \rightarrow \boldsymbol{C}_{*}\left(A, P \otimes_{A} Q\right), \\
\left(m\left[a_{1}|\ldots| a_{n}\right], f\right) \mapsto( & \left(m\left[a_{1}|\ldots| a_{n}\right]\right) \cap f:= \\
& \sum_{p=0}^{n} \pm\left(m \otimes_{A} f\left[a_{1}|\ldots| a_{p}\right]\right)\left[a_{p+1}|\ldots| a_{n}\right] .
\end{aligned}
$$

Here \pm is the Koszul sign $(-1)^{|f|\left(\left|a_{1}\right|+\ldots\left|a_{n}\right|+n\right)}$ [33], proof of Lemma 16.

Let $f: A \rightarrow B$ be a morphism of differential graded algebras and let $N$ be a $B$-bimodule. The linear map $B \otimes_{A} N \rightarrow N, b \otimes n \mapsto b \cdot n$, is a morphism of $B$-bimodules. We call again cap product the composite

$$
\bigodot_{*}(A, B) \otimes \bigodot^{*}(A, N) \stackrel{\cap}{\rightarrow} \bigodot_{*}\left(A, B \otimes_{A} N\right) \rightarrow \bigodot_{*}(A, N) .
$$

In this paper, our goal (Statement 9) is to relate the cap product with $B=A$ to the cap product with $N=B=\mathbb{k}$. 
Statement 9. Let $A$ be an augmented differential graded algebra such that each $A_{i}$ is $\mathbb{k}$-free, $i \in \mathbb{Z}$. Let $c \in \mathrm{HH}_{d}(A, A)$. Denote by $[m] \in \operatorname{Tor}_{d}^{A}(\mathbb{k}, \mathbb{k})$ the image of $c$ by the morphism

$$
\mathrm{HH}_{d}(A, \varepsilon): \mathrm{HH}_{d}(A, A) \rightarrow \mathrm{HH}_{d}(A, \mathbb{k})=\operatorname{Tor}_{d}^{A}(\mathbb{k}, \mathbb{k}) .
$$

Suppose that

- there exists a positive integer $n$ such that $\operatorname{Tor}_{i}^{A}(\mathbb{k}, \mathbb{k})=0$ for all $i \leq-n$ and $i \geq n$,

- each $\operatorname{Tor}_{i}^{A}(\mathbb{k}, \mathbb{k}), i \in \mathbb{Z}$, is of finite type,

- the morphism of right $\operatorname{Ext}_{A}^{*}(\mathbb{k}, \mathbb{k})$-modules

$$
\operatorname{Ext}_{A}^{p}(\mathbb{k}, \mathbb{k}) \stackrel{\cong}{\Longrightarrow} \operatorname{Tor}_{d-p}^{A}(\mathbb{k}, \mathbb{k}), \quad a \mapsto[m] \cap a,
$$

is an isomorphism.

Then for any A-bimodule $N$ the morphism

$$
\mathbb{D}^{-1}: \mathrm{HH}^{p}(A, N) \stackrel{\cong}{\longrightarrow} \mathrm{HH}_{d-p}(A, N), \quad a \mapsto c \cap a,
$$

is also an isomorphism.

This statement is the Eckmann-Hilton or Koszul dual of [33], Proposition 11. In this section we prove this statement if $A$ is connected. But we wonder if it is true in the non-connected case or even for ungraded algebras.

Property 10. Let $B$ and $N$ be two complexes. Consider the natural morphism of complexes $\Theta: B^{\vee} \otimes N \rightarrow \operatorname{Hom}(B, N)$, which sends $\varphi \otimes n$ to the linear map $f: B \rightarrow N$ defined by $f(b):=\varphi(b) n$. Suppose that each $B_{i}$ is $\mathbb{k}$-free. If

1) $B_{i}=0$ for all $i \leq-n$ and $i \geq n$, for some positive integer $n$, and if each $B_{i}$ is of finite type, or if

2) $H_{i}(B)=0$ for all $i \leq-n$ and $i \geq n$, for some positive integer $n$, and if each $H_{i}(B)$ is of finite type,

then $\Theta$ is a homotopy equivalence.

Proof. 1) Since $B$ is bounded, the component of degree $n$ of $\operatorname{Hom}(B, N)$ is the direct sum $\bigoplus_{q \in \mathbb{Z}} \operatorname{Hom}\left(B_{q-n}, N_{q}\right)$. Since $B_{q-n}$ is free of finite type, $\operatorname{Hom}\left(B_{q-n}, N_{q}\right)$ is isomorphic to $B_{q-n}^{\vee} \otimes N_{q}$. Therefore $\Theta$ is an isomorphism.

2) Since $\mathbb{k}$ is a principal ideal domain, the proof of [36], Lemma 5.5.9, shows that there exists a complex $B^{\prime}$ satisfying 1 ) and which is homotopy equivalent to $B$. By its naturality, $\Theta$ is a homotopy equivalence of complexes.

Lemma 11. Statement 9 holds whenever $N$ is a trivial A-bimodule, i.e., $a \cdot n=$ $\varepsilon(a) n=n \cdot a$ for $a \in A$ and $n \in N$. 
Proof. Since $N$ is a trivial $A$-bimodule, the normalized Hochschild chain complex $\bar{C}_{*}(A, N)$ is just the tensor product of complexes $\bar{C}_{*}(A, \mathbb{k}) \otimes N=\bar{B}(A) \otimes N$. (This is also true for the unnormalized Hochschild chain complex, but it is less obvious). And the normalized Hochschild cochain complex $\bar{\ell}^{*}(A, N)$ is just the Hom complex $\operatorname{Hom}\left(\bar{e}_{*}(A, \mathbb{k}), N\right)=\operatorname{Hom}(\bar{B}(A), N)$. Since the augmentation ideal $\bar{A}$ of $A$ is $\mathbb{k}$-free, $\bar{B}(A)$ is also $k$-free. Each $H_{i}(\bar{B}(A))$ is of finite type and $H_{i}(\bar{B}(A))=$ $\operatorname{Tor}_{i}^{A}(\mathbb{k}, \mathbb{k})$ is null if $i \leq-n$ or $i \geq n$. Therefore, by part 2) of Property 10, $\Theta: \bar{B}(A)^{\vee} \otimes N \stackrel{\simeq}{\longrightarrow} \operatorname{Hom}(\bar{B}(A), N)$ is a quasi-isomorphism. A straightforward calculation shows that the following diagram commutes:

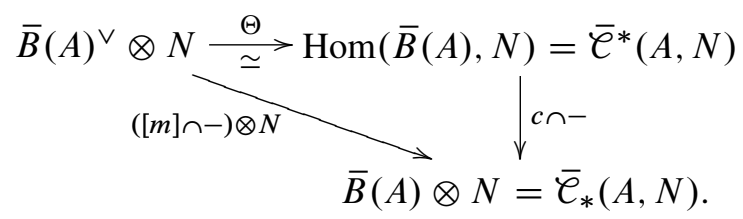

Since $\bar{B}(A)$ is $\mathbb{k}$-free and its dual $\bar{B}(A)^{\vee}$ is torsion-free, by naturality of the Künneth formula [36], Theorem 5.3.3, $([\mathrm{m}] \cap-) \otimes N$ is a quasi-isomorphism. Therefore $c \cap-$ is also a quasi-isomorphism.

Proposition 12. Let $A$ be an augmented differential graded algebra. Let $N$ be an A-bimodule. And let $c \in \mathrm{HH}_{d}(A, A)$ satisfying the hypotheses of Statement 9. For any $k \geq 0$, let $F^{k}:=\bar{A}^{k} \cdot N$. Then taking the inverse limit of the cap product with $c$ induces a quasi-isomorphism of complexes

$$
\lim _{\longleftarrow} c \cap-: \lim _{\longleftarrow} \bigodot^{*}\left(A, N / F^{k}\right) \stackrel{\simeq}{\longrightarrow} \lim _{\longleftarrow} \bigodot_{*}\left(A, N / F^{k}\right) .
$$

Proof. Consider the augmentation ideal $\overline{A^{e}}$ of the enveloping algebra $A^{e}$. For any $k \geq 0$, let $\bar{A}^{e}$ be the image of the iterated tensor product $\bar{A}^{\otimes k}$ by the iterated multiplication of $A^{e}, \mu:\left(A^{e}\right)^{\otimes k} \rightarrow A^{e}$, and let $F^{k}$ be the image of $\bar{A}^{e}{ }^{k} \otimes N$ by the action $A^{e} \otimes N \rightarrow N$.

The $F^{k}$ form a decreasing filtration of sub- $A$-bimodules and subcomplexes of $N$. Since $F^{k} / F^{k+1}$ is a trivial $A$-bimodule, by Lemma 11, the morphism of complexes

$$
\ell^{*}\left(A, F^{k} / F^{k+1}\right) \stackrel{\simeq}{\longrightarrow} \ell_{*}\left(A, F^{k} / F^{k+1}\right), \quad a \mapsto c \cap a,
$$

is a quasi-isomorphism. By Noether's theorem, we have the short exact sequences of $A$-bimodules

$$
0 \rightarrow F^{k} / F^{k+1} \rightarrow N / F^{k+1} \rightarrow N / F^{k} \rightarrow 0 .
$$

Since $T(\mathrm{~s} A)$ is $\mathbb{k}$-free, the functors $\operatorname{Hom}_{\mathbb{k}}(T(\mathrm{~s} A),-)$ and $-\bigotimes_{\mathbb{k}} T(\mathrm{~s} A)$ preserve short exact sequences. Therefore consider the morphism of short exact sequences of 
complexes induced by the cap product with $c$ :

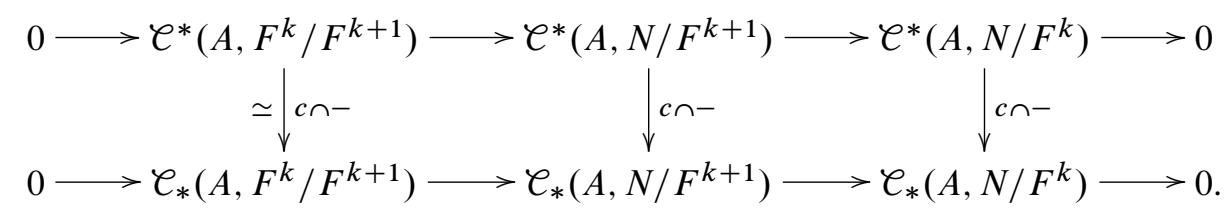

Using the long exact sequences associated and the five lemma, by induction on $k$, we obtain that the morphism of complexes

$$
\ell^{*}\left(A, N / F^{k}\right) \stackrel{\simeq}{\longrightarrow} \ell_{*}\left(A, N / F^{k}\right), \quad a \mapsto c \cap a,
$$

is a quasi-isomorphism for all $k \geq 0$.

The two towers of complexes

$$
\begin{aligned}
& \cdots \rightarrow \ell^{*}\left(A, N / F^{k+1}\right) \rightarrow \ell^{*}\left(A, N / F^{k}\right) \rightarrow \cdots, \\
& \cdots \rightarrow \ell_{*}\left(A, N / F^{k+1}\right) \rightarrow \ell_{*}\left(A, N / F^{k}\right) \rightarrow \cdots
\end{aligned}
$$

satisfy the trivial Mittag-Leffler condition since all the maps in the two towers are onto. Therefore by naturality of [40], Theorem 3.5.8, for each $p \in \mathbb{Z}$, we have the morphism of short exact sequences induced by the cap product with $c$ :

$$
\begin{aligned}
& \lim ^{1} \mathrm{HH}^{p-1}\left(A, N / F^{k}\right) \longrightarrow H^{p} \lim \ell^{*}\left(A, N / F^{k}\right) \longrightarrow \lim _{\sim} \operatorname{HH}^{p}\left(A, N / F^{k}\right)
\end{aligned}
$$

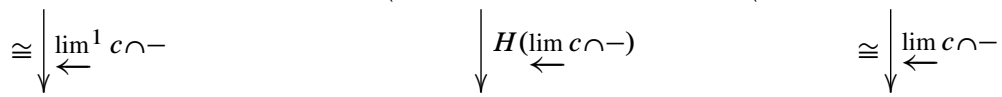

$$
\begin{aligned}
& \lim ^{1} \mathrm{HH}_{d+1-p}\left(A, N / F^{k}\right) \rightarrow H_{d-p} \lim _{\longleftarrow} \ell_{*}\left(A, N / F^{k}\right) \rightarrow \underset{\longleftarrow}{\lim } \operatorname{HH}_{d-p}\left(A, N / F^{k}\right) .
\end{aligned}
$$

Using the five lemma again, we obtain that the middle morphism

$$
H\left(\lim _{\longleftarrow} c \cap-\right): H^{p} \lim _{\longleftarrow} \bigodot^{*}\left(A, N / F^{k}\right) \rightarrow H_{d-p} \lim _{\longleftarrow} \ell_{*}\left(A, N / F^{k}\right)
$$

is an isomorphism.

Corollary 13. Statement 9 is true if $A$ and $N$ are non-negatively lower graded and $H_{0}(\varepsilon): H_{0}(A) \stackrel{\cong}{\cong}$ is an isomorphism.

Proof. Case 1: We first suppose that $\varepsilon: A_{0} \cong \mathbb{\cong}$ is an isomorphism. Then $\bar{A}^{k}$ is concentrated in degrees $\geq k$. Therefore $F^{k}$ and $\ell_{*}\left(A, F^{k}\right)$ are also concentrated in degrees $\geq k$. This means that for $n<k$ their components of degree $n,\left(F^{k}\right)_{n}$ and $\left[\mathcal{C}_{*}\left(A, F^{k}\right)\right]_{n}$, are trivial. Therefore the tower in degree $n$

$$
\cdots \rightarrow\left(N / F^{k+1}\right)_{n} \rightarrow\left(N / F^{k}\right)_{n} \rightarrow \cdots
$$

is constant and equal to $N_{n}$ for $k>n$. This implies that $N_{n}=\underset{\lim }{\longleftarrow}\left(N / F^{k}\right)_{n}$. Therefore as complexes and as $A$-bimodule, $N=\lim _{\longleftarrow} N / F^{k}$. 
Since $\ell_{*}\left(A, N / F^{k}\right)$ is the quotient $\ell_{*}(A, N) / \ell_{*}\left(A, F^{k}\right)$, we also have that, as complexes,

$$
e_{*}(A, N)=\lim _{\leftarrow} e_{*}\left(A, N / F^{k}\right)
$$

The functor $\ell^{*}(A,-)$ from (differential) $A$-bimodules to complexes is a right adjoint (to the functor $B(A ; A ; A) \otimes-)$. Therefore $\complement^{*}(A,-)$ preserves inverse limits. Since $N=\lim N / F^{k}$ in the category of (differential) $A$-bimodules, we obtain that as complex

$$
\ell^{*}(A, N)=\ell^{*}\left(A, \lim _{\longleftarrow} N / F^{k}\right)=\lim _{\longleftarrow} \ell^{*}\left(A, N / F^{k}\right) .
$$

Since for any $k \geq 0$ the square

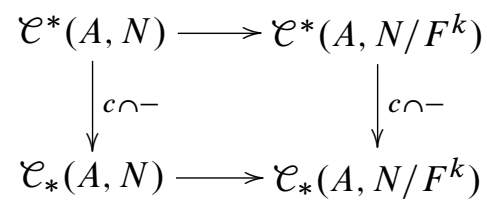

commutes, the quasi-isomorphism

$$
\lim _{\longleftarrow} c \cap-: \lim _{\longleftarrow} e^{*}\left(A, N / F^{k}\right) \stackrel{\simeq}{\longleftarrow} \lim _{\longleftarrow} C_{*}\left(A, N / F^{k}\right)
$$

given by Proposition 12 coincides with $c \cap-: \complement^{*}(A, N) \rightarrow \bigodot_{*}(A, N)$.

Case 2: Now we only suppose that $H_{0}(\varepsilon): H_{0}(A) \stackrel{\cong}{\longrightarrow} \mathrm{k}$ is an isomorphism. Let $\tilde{A}$ be the graded $\mathbb{k}$-module defined by $\tilde{A}_{0}=\mathbb{k}, \tilde{A}_{1}=\operatorname{ker} d: A_{1} \rightarrow A_{0}, \tilde{A}_{n}=A_{n}$ for $n \geq 2$ (compare with the upper graded version in [11], p. 184). Clearly $\tilde{A}$ is a k-free differential graded subalgebra of $A$ and the inclusion $j: \tilde{A} \stackrel{\simeq}{\hookrightarrow} A$ is a quasiisomorphism since $\operatorname{im}\left(d: A_{1} \rightarrow A_{0}\right)$ is equal to $\overline{A_{0}}$.

Since the augmentation ideals of $A$ and $\tilde{A}, \bar{A}$ and $\overline{\tilde{A}}$, are $\mathbb{k}$-free and non-negatively lower graded, the three morphisms $\mathrm{HH}_{*}(j, N): \mathrm{HH}_{*}(\tilde{A}, N) \stackrel{\cong}{\cong} \mathrm{HH}_{*}(A, N)$, $\mathrm{HH}^{*}(j, N): \mathrm{HH}^{*}(A, N) \stackrel{\cong}{\cong} \mathrm{HH}^{*}(\tilde{A}, N), \mathrm{HH}_{*}(j, j): \mathrm{HH}_{*}(\tilde{A}, \tilde{A}) \stackrel{\cong}{\longrightarrow} \mathrm{HH}_{*}(A, A)$ are all isomorphisms by [28], 5.3.5, or [10], 4.3 (iii). Let $\tilde{c} \in \mathrm{HH}_{d}(\tilde{A}, \tilde{A})$ such that $\mathrm{HH}_{d}(j, j)(\tilde{c})=c$. Using the definition of the cap product, it is straightforward to check that the square

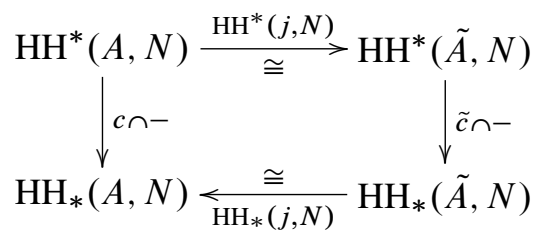

commutes. Let $[\tilde{m}] \in \operatorname{Tor}_{d}^{\tilde{A}}(\mathbb{k}, \mathbb{k})$ such that $\operatorname{Tor}_{d}^{j}(\mathbb{k}, \mathbb{k})([\tilde{m}])=[m]$. When $N=\mathbb{k}$, 
the previous square specializes to the commutative square

$$
\begin{aligned}
& \operatorname{Ext}_{A}^{*}(\mathbb{k}, \mathbb{k}) \stackrel{\operatorname{Ext}_{-}^{*} j(\mathbb{k}, \mathbb{k})}{\cong} \operatorname{Ext}_{\tilde{A}}^{*}(\mathbb{k}, \mathbb{k}) \\
& {[m] \cap-\mid \cong} \\
& \operatorname{Tor}_{*}^{A}(\mathbb{k}, \mathbb{k}) \underset{\operatorname{Tor}_{*}^{j}(\mathbb{k}, \mathbb{k})}{\cong} \operatorname{Tor}_{*}^{\tilde{A}}(\mathbb{k}, \mathbb{k}) .
\end{aligned}
$$

By hypothesis, $[m] \cap-$ is an isomorphism. Therefore $[\tilde{m}] \cap-$ is also an isomorphism. Since $\tilde{A}_{0}=\mathbb{k}$, we have seen in case 1 that

$$
\tilde{c} \cap-: \operatorname{HH}^{*}(\tilde{A}, N) \stackrel{\cong}{\cong} \mathrm{HH}_{*}(\tilde{A}, N)
$$

is an isomorphism. Therefore

$$
c \cap-: \mathrm{HH}^{*}(A, N) \stackrel{\cong}{\cong} \mathrm{HH}_{*}(A, N)
$$

is also an isomorphism.

Corollary 14. Statement 9 is true if $A$ and $N$ are non-negatively upper graded, $H^{0}(\varepsilon): H^{0}(A) \stackrel{\cong}{\cong} \mathbb{k}$ is an isomorphism and $\mathbb{k}$ is a field.

Proof. Case 1: We first suppose that $\varepsilon: A^{0} \stackrel{\cong}{\longrightarrow} \mathbb{k}$ is an isomorphism. Since $T(\mathrm{~s} A)$ has non-trivial elements of negative degrees, we need to use the normalized Hochschild chain and cochain complexes $\bar{\ell}_{*}$ and $\bar{\ell}^{*}$ instead of the unnormalized $\mathcal{C}_{*}$ and $\ell^{*}$. Now the proof is the same as in case 1 of the proof of Corollary 13.

Case 2: Now we only suppose that $H^{0}(\varepsilon): H^{0}(A) \stackrel{\cong}{\longrightarrow} \mathbb{k}$ is an isomorphism. Since $\mathbb{k}$ is a field, by [11], p. 184, there exists a differential graded algebra $\tilde{A}$, nonnegatively upper graded, equipped with a quasi-isomorphism $j: \tilde{A} \stackrel{\simeq}{\longrightarrow} A$ such that $\tilde{A}^{0}=\mathbb{k}$. Now the rest of the proof is exactly the same as in case 2 of the proof of Corollary 13 .

\section{Comparison of the cap products in Hochschild and group (co)homology}

Let $G$ be a discrete group. Let $M$ and $N$ be two $\mathbb{k}[G]$-bimodules. Let $\eta: \mathbb{k} \rightarrow \mathbb{k}[G]$ be the unit map. Let $E: \mathbb{k}[G] \rightarrow \mathbb{k}\left[G \times G^{\mathrm{op}}\right]$ be the morphism of algebras mapping $g$ to $\left(g, g^{-1}\right)$. Let

$$
\tilde{\eta}: \mathbb{k}\left[G \times G^{\mathrm{op}}\right] \otimes_{\mathbb{k}[G]} \mathbb{k} \rightarrow \mathbb{k}[G]
$$

be the unique morphism of left $\mathbb{k}\left[G \times G^{\mathrm{op}}\right]$-modules extending $\eta$. Since $\mathbb{k}\left[G \times G^{\mathrm{op}}\right]$ is flat as left $\mathbb{k}[G]$-module via $E$ and since $\tilde{\eta}$ is an isomorphism, by Eckmann-Schapiro 
[22], Chapter IV, Proposition 12.2, we obtain the well-known isomorphisms between Hochschild (co)homology and group (co)homology:

$$
\begin{aligned}
& \operatorname{Ext}_{E}^{*}(\eta, N): \mathrm{HH}^{*}(\mathbb{k}[G], N)=\operatorname{Ext}_{\mathbb{k}\left[G \times G^{\mathrm{op}}\right]}^{*}(\mathbb{k}[G], N) \\
& \stackrel{\cong}{\longrightarrow} \operatorname{Ext}_{\mathbb{k}[G]}^{*}(\mathbb{k}, \tilde{N})=H^{*}(G, \tilde{N}) .
\end{aligned}
$$

and

$$
\begin{aligned}
\operatorname{Tor}_{*}^{E}(M, \eta): H_{*}(G, \tilde{M})= & \operatorname{Tor}_{*}^{\mathbb{k}[G]}(\tilde{M}, \mathbb{k}) \\
& \stackrel{\cong}{\longrightarrow} \operatorname{Tor}_{\mathbb{k}\left[G \times G^{\mathrm{op}]}\right.}^{*}(M, \mathbb{k}[G])=\mathrm{HH}_{*}(\mathbb{k}[G], M) .
\end{aligned}
$$

Here $\tilde{M}$ and $\tilde{N}$ denote the $\mathbb{k}[G]$-modules obtained by restriction of scalar via $E$. Note that we regard any left $\mathbb{k}[G]$-module as a right $\mathbb{k}[G]$-module via $g \mapsto g^{-1}[4]$, p. 55.

Proposition 15. Observe that the canonical surjection

$$
q: \tilde{M} \otimes \tilde{N} \rightarrow \widehat{M \otimes_{\mathbb{k}[G]} N}
$$

is a morphism of $\mathbb{k}[G]$-modules since $q\left(g m g^{-1} \otimes g n g^{-1}\right)=g m \otimes n g^{-1}$.

i) Cup product $\cup$ in Hochschild cohomology versus cup product in group cohomology (slight extension of [35], Proposition 3.1). The following diagram commutes:

$$
\begin{aligned}
& \mathrm{HH}^{*}(\mathbb{k}[G], M) \otimes \mathrm{HH}^{*}(\mathbb{k}[G], N) \longrightarrow \mathrm{HH}^{*}\left(\mathbb{k}[G], M \otimes_{\mathbb{k}[G]} N\right) \\
& \begin{array}{l}
\downarrow \operatorname{Ext}_{E}^{*}(\eta, M) \otimes \operatorname{Ext}_{E}^{*}(\eta, N) \quad \operatorname{Ext}_{E}^{*}\left(\eta, M \otimes_{\mathbb{k}[G]} N\right) \downarrow \\
\otimes H^{*}(G, \tilde{N}) \longrightarrow H^{*}(G, \tilde{M} \otimes \tilde{N})_{H^{*}(G, q)} H^{*}\left(G, \widehat{M \otimes_{\mathbb{k}[G]} N}\right) .
\end{array}
\end{aligned}
$$

ii) Cap products $\cap$. The following diagram commutes:

$$
\begin{aligned}
& \mathrm{HH}_{*}(\mathbb{k}[G], M) \otimes \mathrm{HH}^{*}(\mathbb{k}[G], N) \longrightarrow \mathrm{HH}_{*}\left(\mathbb{k}[G], M \otimes_{\mathbb{k}[G]} N\right) \\
& \begin{array}{l}
\uparrow \operatorname{Tor}_{*}^{E}(M, \eta) \otimes \operatorname{Ext}_{E}^{*}(\eta, N)^{-1} \quad \operatorname{Tor}_{*}^{E}\left(M \otimes_{\mathbb{k}[G]} N, \eta\right) \uparrow \\
\otimes H^{*}(G, \tilde{N}) \underset{\cap}{\longrightarrow} H_{*}(G, \tilde{M} \otimes \tilde{N})_{H_{*}(G, q)} H_{*}\left(G, \widehat{M \otimes_{\mathbb{k}[G]} N}\right) .
\end{array}
\end{aligned}
$$

Remark 16. In the case $N=\mathbb{k}[G]$ [35], (3.3), the morphism of $\mathbb{k}[G]$-modules $q: \tilde{M} \otimes \mathbb{k}\left[\tilde{[G]} \rightarrow \widehat{M \otimes_{\mathbb{k}[G]} \mathbb{k}[G]} \cong \tilde{M}\right.$ is simply the action $m \otimes g \mapsto m \cdot g$.

In the case $M=N=\mathbb{k}[G]$ the diagram i) in Proposition 15 means that

$$
\operatorname{Ext}_{E}^{*}(\eta, \mathbb{k}[G]): \operatorname{HH}^{*}(\mathbb{k}[G], \mathbb{k}[G]) \rightarrow H^{*}(G, \mathbb{k}[G])
$$

is a morphism of graded algebras.

In the case $N=\mathbb{k}[G]$ the diagram ii) means that

$$
\operatorname{Tor}_{*}^{E}(M, \eta): H_{*}(G, \tilde{M}) \rightarrow \mathrm{HH}_{*}(\mathbb{k}[G], M)
$$


is a morphism of right $\mathrm{HH}^{*}(\mathbb{k}[G], \mathbb{k}[G])$-modules:

$$
\operatorname{Tor}_{*}^{E}(\eta, \mathbb{k}[G])\left(\alpha \cap \operatorname{Ext}_{E}^{*}(\eta, \mathbb{k}[G])(\varphi)\right)=\operatorname{Tor}_{*}^{E}(\eta, \mathbb{k}[G])(\alpha) \cap \varphi
$$

for any $\alpha \in H_{*}(G, \tilde{M})$ and any $\varphi \in \mathrm{HH}^{*}(\mathbb{k}[G], \mathbb{k}[G])$.

Proof. Siegel and Witherspoon [35], Proposition 3.1, proved i) using that, for any projective resolution $P$ of $\mathbb{k}$ as left $\mathbb{k}[G]$-modules,

$$
X:=\mathbb{k}\left[G \times G^{\mathrm{op}}\right] \otimes_{\mathbb{k}[G]} P
$$

is a projective resolution of $\mathbb{k}[G]$ as $\mathbb{k}[G]$-bimodules. Let $\iota: P \hookrightarrow \tilde{X}$ the left $\mathbb{k}[G]$ linear map defined by $\iota(x)=(1,1) \otimes x$. Using that

$$
\operatorname{Hom}_{E}(\iota, N): \operatorname{Hom}_{\mathbb{k}\left[G \times G^{\mathrm{op}}\right]}(X, N) \stackrel{\cong}{\longrightarrow} \operatorname{Hom}_{\mathbb{k}[G]}(P, \tilde{N})
$$

is an isomorphism of complexes inducing $\operatorname{Ext}_{E}^{*}(\eta, N)$ and that

$$
M \otimes_{E} \iota: \tilde{M} \otimes_{\mathbb{k}[G]} P \cong M \otimes_{\mathbb{k}\left[G \times G^{\mathrm{op}}\right]} X
$$

is an isomorphism of complexes inducing $\operatorname{Tor}_{*}^{E}(M, \eta)$, Siegel and Witherspoon [35], Proposition 3.1 , proved i). It is possible to prove ii) in a similar way.

We find it simpler to give a proof of ii) using the bar resolution.

Let $\iota: B(\mathbb{k}[G] ; \mathbb{k}[G] ; \mathbb{k}) \rightarrow \widehat{B(\mathbb{k}[G] ; \mathbb{k}[G] ; \mathbb{k}[G])}$ be the linear map defined by

$$
\iota\left(g_{0}\left[g_{1}|\ldots| g_{n}\right]\right)=g_{0}\left[g_{1}|\ldots| g_{n}\right] g_{n}^{-1} \ldots g_{0}^{-1} .
$$

Obviously $\iota$ fits into the commutative diagram of left $\mathbb{k}[G]$-modules

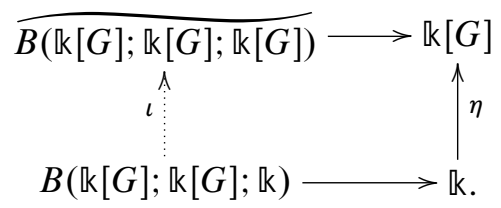

By an easy computation, $\iota$ is a morphism of complexes. Thus $\operatorname{Hom}_{E}(\iota, N)$ is a morphism of complexes from $\mathcal{C}^{*}(\mathbb{k}[G], N) \cong\left(\operatorname{Hom}_{\mathbb{k}}\left[G \times G^{\mathrm{op}}\right](B(\mathbb{k}[G] ; \mathbb{k}[G] ; \mathbb{k}[G]), N)\right.$ to $\operatorname{Hom}_{\mathbb{k}[G]}(B(\mathbb{k}[G] ; \mathbb{k}[G] ; \mathbb{k}), \tilde{N})$. Inducing $\operatorname{Ext}_{E}^{*}(\eta, N)$ and $M \otimes_{E} \iota$ is a morphism of complexes from

$$
B(\tilde{M} ; \mathbb{k}[G] ; \mathbb{k}) \cong \tilde{M} \otimes_{\mathbb{k}[G]} B(\mathbb{k}[G] ; \mathbb{k}[G] ; \mathbb{k})
$$

to

$$
M \otimes_{\mathbb{k}\left[G \times G^{\mathrm{op}}\right]} B(\mathbb{k}[G] ; \mathbb{k}[G] ; \mathbb{k}[G]) \cong \mathcal{C}_{*}(\mathbb{k}[G], M),
$$

inducing $\operatorname{Tor}_{*}^{E}(M, \eta)$. Explicitly $M \otimes_{E} \iota$ is the morphism of complexes

$$
B(\tilde{M} ; \mathbb{k}[G] ; \mathbb{k}) \rightarrow \bigodot_{*}(\mathbb{k}[G], M)
$$


defined by [14], (2.20),

$$
\xi\left(m\left[g_{1}|\ldots| g_{n}\right]=g_{n}^{-1} \ldots g_{1}^{-1} m\left[g_{1}|\ldots| g_{n}\right] .\right.
$$

And $\operatorname{Hom}_{E}(\iota, N): e^{n}(\mathbb{k}[G], N) \rightarrow \operatorname{Hom}\left(\mathbb{k}[G]^{\otimes n}, \tilde{N}\right)$ is the linear map $\xi$ mapping $\varphi \in \mathcal{C}^{n}(\mathbb{k}[G], N)$ to the linear map $\xi(\varphi): \mathbb{k}[G]^{\otimes n} \rightarrow \tilde{N}$ defined by

$$
\xi(\varphi)\left(\left[g_{1}|\ldots| g_{n}\right]\right)=\varphi\left(\left[g_{1}|\ldots| g_{n}\right]\right) g_{n}^{-1} \ldots g_{1}^{-1} .
$$

Both $M \otimes_{E} \iota$ and $\operatorname{Hom}_{E}(\iota, N)$ are in fact isomorphisms of complexes. The inverse of $M \otimes_{E} \iota$ is the morphism of complexes $\Phi: \digamma_{*}(\mathbb{k}[G], M) \rightarrow B(\tilde{M} ; \mathbb{k}[G] ; \mathbb{k})$ defined by [28], 7.4.2.1,

$$
\Phi\left(m\left[g_{1}|\ldots| g_{n}\right]\right)=g_{1} \ldots g_{n} m\left[g_{1}|\ldots| g_{n}\right] .
$$

Let $F$ be any projective resolution of $\mathbb{k}$ as left $\mathbb{k}[G]$-module. Let $P$ and $Q$ be two $\mathbb{k}[G]$-modules. The cap product in group cohomology is the composite [4], p. 113, denoted $\cap$,

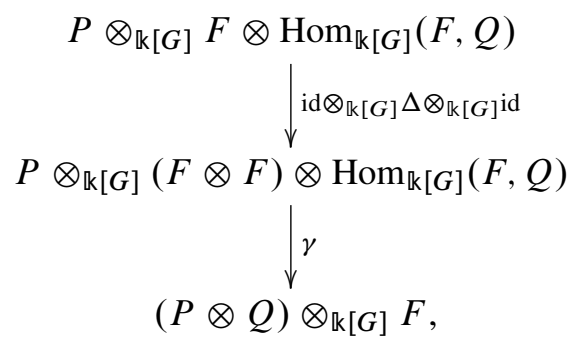

where $\gamma(a \otimes x \otimes y \otimes u)=(-1)^{|u||x|+|u||y|} a \otimes u(x) \otimes y$ and $\Delta$ is a diagonal approximation. In case $F$ is the bar resolution $B(\mathbb{k}[G] ; \mathbb{k}[G] ; \mathbb{k})$, one can take $\Delta$ to be the Alexander-Whitney map

$$
A W: B(\mathbb{k}[G] ; \mathbb{k}[G] ; \mathbb{k}) \rightarrow B(\mathbb{k}[G] ; \mathbb{k}[G] ; \mathbb{k}) \otimes B(\mathbb{k}[G] ; \mathbb{k}[G] ; \mathbb{k})
$$

defined by [4], (1.4), p. 108:

$$
A W\left(g_{0}\left[g_{1}|\ldots| g_{n}\right]\right)=\sum_{p=0}^{n} g_{0}\left[g_{1}|\ldots| g_{p}\right] \otimes g_{0} \ldots g_{p}\left[g_{p+1}|\ldots| g_{n}\right] .
$$

Therefore the cap product

$$
\cap: B(P ; \mathbb{k}[G] ; \mathbb{k}) \otimes \operatorname{Hom}(B(\mathbb{k}[G]), Q), d \rightarrow B(P \otimes Q ; \mathbb{k}[G] ; \mathbb{k})
$$

is the morphism of complexes mapping $m\left[g_{1}|\ldots| g_{n}\right] \otimes u: G^{p} \rightarrow Q$ to $m \cdot g_{1} \ldots g_{p} \otimes$ $u\left(g_{1}, \ldots, g_{p}\right) \cdot g_{1} \ldots g_{p}\left[g_{p+1}|\ldots| g_{n}\right]$. Using the explicit formula (7) for the cap product in Hochschild cohomology, it is easy to check that the diagram

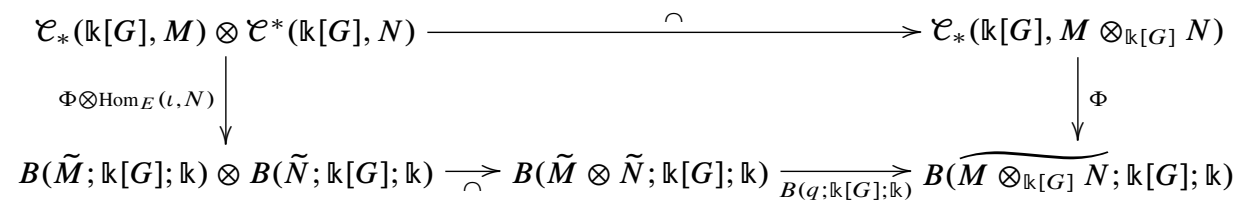

commutes. By applying homology, ii) is proved. 
Definition 18 ([28], 7.4 .5 when $z=1)$. Let $\sigma: B(\mathbb{k}[G]) \hookrightarrow \mathcal{C}_{*}(\mathbb{k}[G], \mathbb{k}[G])$ be the linear map defined by

$$
\sigma\left(\left[g_{1}|\ldots| g_{n}\right]\right)=g_{n}^{-1} \ldots g_{1}^{-1}\left[g_{1}|\ldots| g_{n}\right] .
$$

Property 19. i) The map $\sigma$ is a morphism of cyclic modules ([28], 7.4.5 when $z=1$ ).

ii) The morphism $\sigma$ of complexes coincides with the composite

$$
B(\mathbb{k}[G]) \stackrel{B(\eta ; \mathbb{k}[G] ; \mathbb{k})}{\longrightarrow} B(\widetilde{\mathbb{k}[G]} ; \mathbb{k}[G] ; \mathbb{k}) \stackrel{\xi}{\cong} \bigodot_{*}(\mathbb{k}[G] ; \mathbb{k}[G]) .
$$

Here $\xi$ is the isomorphism of complexes defined by (17). Note that the unit map $\eta: \mathbb{k} \rightarrow \widetilde{\mathbb{k}[G]}$ is a morphism of $\mathbb{k}[G]$-modules.

iii) In particular, in homology, $\sigma$ coincides with

$$
\operatorname{Tor}^{E}(\eta, \eta): H_{*}(G ; \mathbb{k}) \rightarrow \mathrm{HH}_{*}(\mathbb{k}[G] ; \mathbb{k}[G]) .
$$

iv) The map $\sigma$ is a section of

$$
\boldsymbol{C}_{*}(\mathbb{k}[G], \varepsilon): \boldsymbol{C}_{*}(\mathbb{k}[G], \mathbb{k}[G]) \rightarrow \boldsymbol{C}_{*}(\mathbb{k}[G], \mathbb{k})=B(\mathbb{k}[G]) .
$$

Corollary 20. Let $G$ be any discrete group, $N$ be a $\mathbb{k}[G]$-bimodule, $\sigma: H_{*}(G ; \mathbb{k}) \rightarrow$ $\mathrm{HH}_{*}(\mathbb{k}[G] ; \mathbb{k}[G])$ be the section of $\mathrm{HH}_{*}(\mathbb{k}[G], \varepsilon): \mathrm{HH}_{*}(\mathbb{k}[G], \mathbb{k}[G]) \rightarrow H_{*}(G, \mathbb{k})$ given in Definition 18. Let $z \in H_{d}(G, \mathbb{k})$ be any element in group homology. Then the square

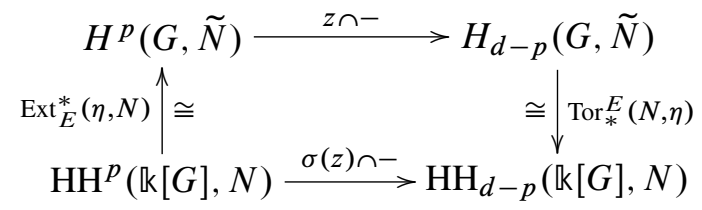

commutes.

Proof. Consider

$$
\begin{aligned}
& \mathrm{HH}_{*}(\mathbb{k}[G], \mathbb{k}[G]) \otimes \mathrm{HH}^{*}(\mathbb{k}[G], N) \longrightarrow \mathrm{HH}_{*}\left(\mathbb{k}[G], \mathbb{k}[G] \otimes_{\mathbb{k}[G]} N\right) \\
& \uparrow \operatorname{Tor}_{*}^{E}(\mathbb{k}[G], \eta) \otimes \operatorname{Ext}_{E}^{*}(\eta, N)^{-1} \quad \operatorname{Tor}_{*}^{E}(N, \eta) \uparrow \\
& H_{*}(G, \widetilde{\mathbb{k}[G]}) \otimes H^{*}(G, \tilde{N}) \underset{\cap}{\longrightarrow} H_{*}(G, \widetilde{\mathbb{k}[G]} \otimes \tilde{N}) \underset{H_{*}(G, q)}{\longrightarrow} H_{*}(G, \tilde{N})
\end{aligned}
$$

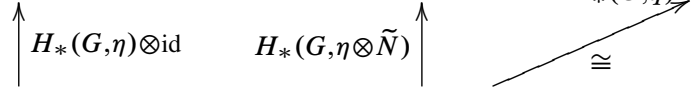

$$
\begin{aligned}
& H_{*}(G, \mathbb{k}) \otimes H^{*}(G, \tilde{N}) \longrightarrow H_{*}(G, \mathbb{k} \otimes \tilde{N}) \text {. }
\end{aligned}
$$

The top rectangle commutes by ii) of Proposition 15 in the case $M=\mathbb{k}[G]$. The bottom square commutes by naturality of the cap product in group (co)homology 
with respect to the morphism of $\mathbb{k}[G]$-modules $\eta: \mathbb{k} \rightarrow \widetilde{\mathbb{k}[G]}$. The bottom triangle commutes by functoriality of $H_{*}(G,-)$. By ii) or iii) of Property 19 , the vertical composite is

$\sigma \otimes \operatorname{Ext}_{E}^{*}(\eta, N)^{-1}: H_{*}(G, \mathbb{k}) \otimes H^{*}(G, \tilde{N}) \rightarrow \mathrm{HH}_{*}(\mathbb{k}[G], \mathbb{k}[G]) \otimes \mathrm{HH}^{*}(\mathbb{k}[G], N)$.

\section{A new definition of Batalin-Vilkovisky algebras}

Definition 21. A Gerstenhaber algebra is a commutative graded algebra $A$ equipped with a linear map $\{-,-\}: A_{i} \otimes A_{j} \rightarrow A_{i+j+1}$ of degree 1 such that the following holds:

a) The bracket $\{-,-\}$ gives $A$ a structure of graded Lie algebra of degree 1 . This means that, for each $a, b$ and $c \in A$,

$$
\{a, b\}=-(-1)^{(|a|+1)(|b|+1)}\{b, a\}
$$

and

$$
\{a,\{b, c\}\}=\{\{a, b\}, c\}+(-1)^{(|a|+1)(|b|+1)}\{b,\{a, c\}\} .
$$

b) The product and the Lie bracket satisfy the Poisson relation

$$
\{a, b c\}=\{a, b\} c+(-1)^{(|a|+1)|b|} b\{a, c\} .
$$

Definition 23. A Batalin-Vilkovisky algebra is a Gerstenhaber algebra $A$ equipped with a degree 1 linear map $\Delta: A_{i} \rightarrow A_{i+1}$ such that $\Delta \circ \Delta=0$ and the bracket is given by

$$
\{a, b\}=(-1)^{|a|}\left(\Delta(a \cup b)-(\Delta a) \cup b-(-1)^{|a|} a \cup(\Delta b)\right)
$$

for $a$ and $b \in A$.

Remark 25. In (24) a sign (here the sign chosen is (-1) ${ }^{|a|}$ ) is needed (see [25], (1.6), or [17], beginning of the proof of Proposition 1.2) since the Lie bracket of degree +1 is graded antisymmetric (eq. (22)), while the associative product is graded commutative. Therefore in the definition of Batalin-Vilkovisky algebra in [18], Theorem 3.4.3 (ii), and in [26], p. 1, there is a sign problem.

The following characterization of Batalin-Vilkovisky algebras was proved by Koszul and rediscovered by Getzler and by Penkava and Schwarz.

Proposition 26 ([25], p. 3, [17], Proposition 1.2, [34]). Let $A$ be a commutative graded algebra $A$ equipped with an operator $\Delta: A_{i} \rightarrow A_{i+1}$ of degree 1 such that $\Delta \circ \Delta=0$. Consider the bracket $\{$,$\} of degree +1$ defined by

$$
\{a, b\}=(-1)^{|a|}\left(\Delta(a b)-(\Delta a) b-(-1)^{|a|} a(\Delta b)\right)
$$


for any $a, b \in A$. Then $A$ is a Batalin-Vilkovisky algebra if and only if $\Delta$ is a differential operator of degree $\leq 2$, which means that, for $a, b$ and $c \in A$,

$$
\begin{aligned}
\Delta(a b c)= & \Delta(a b) c+(-1)^{|a|} a \Delta(b c)+(-1)^{(|a|-1)|b|} b \Delta(a c) \\
& -(\Delta a) b c-(-1)^{|a|} a(\Delta b) c-(-1)^{|a|+|b|} a b(\Delta c) .
\end{aligned}
$$

Note that until now, in this section it is not necessary that the algebras have a unit. Now if the algebras have a unit, we give a new characterization of the Batalin-Vilkovisky algebra. One implication of this new characterization is inspired by Ginzburg's proof of Proposition 32. As we will recall in the proof of Theorem 66, the converse of this characterization is due to [24], "the restriction of this derived bracket to $A$ is the BV-bracket", p. 1270.

Proposition 28. Let $A$ be a Gerstenhaber algebra $A$ equipped with an operator $\Delta: A \rightarrow A$ of degree 1 such that $\Delta \circ \Delta=0$. For any $a \in A$, denote by $l_{a}: A \rightarrow A$ the left multiplication by $a$, explicitly $l_{a}(b)=a b, b \in A$. Denote by $[f, g]=$ $f \circ g-(-1)^{|f||g|} g \circ g$ the graded commutator of two endomorphisms $f: A \rightarrow A$ and $g: A \rightarrow A$. Then $A$ is a Batalin-Vilkovisky algebra if and only if

$$
l_{\{a, b\}}=-\left[\left[l_{a}, \Delta\right], l_{b}\right] \text { and } \Delta(1)=0
$$

for $a, b \in A$.

Proof. For $a$ and $b \in A$,

$$
\begin{aligned}
& {\left[\left[l_{a}, \Delta\right], l_{b}\right]=}\left(l_{a} \circ \Delta-(-1)^{\mid a} \Delta \circ l_{a}\right) \circ l_{b}-(-1)^{|b|(|a|+1)} l_{b} \\
& \circ\left(l_{a} \circ \Delta-(-1)^{\mid a} \Delta \circ l_{a}\right) \\
&= l_{a} \circ \Delta \circ l_{b}-(-1)^{|a|} \Delta \circ l_{a b}-(-1)^{|b|} l_{a b} \\
& \circ \Delta+(-1)^{|b|(|a|+1)+|a|} l_{b} \circ \Delta \circ l_{a} .
\end{aligned}
$$

Therefore by applying this equality of operators to $c \in A$ we have

$$
\begin{aligned}
-(-1)^{|a|}\left[\left[l_{a}, \Delta\right], l_{b}\right](c)= & -(-1)^{|a|} a \Delta(b c)+\Delta(a b c) \\
& +(-1)^{|a|+|b|} a b \Delta(c)-(-1)^{|b|(|a|+1)} b \Delta(a c) .
\end{aligned}
$$

Suppose that $A$ is a Batalin-Vilkovisky algebra. By Proposition 26, using (29), we obtain that

$$
-(-1)^{|a|}\left[\left[l_{a}, \Delta\right], l_{b}\right](c)=\Delta(a b) c-(\Delta a) b c-(-1)^{|a|} a(\Delta b) c=(-1)^{|a|}\{a, b\} c .
$$

Therefore $-\left[\left[l_{a}, \Delta\right], l_{b}\right]=l_{\{a, b\}}$. In the case $a=b=c=1$, eq. (27) gives $\Delta(1)=3 \Delta(1)-3 \Delta(1)=0$.

Conversely, suppose that $\Delta(1)=0$ and $l_{\{a, b\}}=-\left[\left[l_{a}, \Delta\right], l_{b}\right]$. Then using (29) we have

$$
\{a, b\}=l_{\{a, b\}}(1)=(-1)^{|a|}\left(-(-1)^{|a|} a \Delta(b)+\Delta(a b)+0-(\Delta a) b\right) .
$$

Therefore, by Definition 23, $A$ is a Batalin-Vilkovisky algebra. 


\section{Batalin-Vilkovisky algebra structures on Hochschild cohomology}

Let $A$ be a differential graded algebra. The cap product defined in Section 3,

$$
\mathrm{HH}_{*}(A, A) \otimes \mathrm{HH}^{*}(A, A) \stackrel{\bigcap}{\rightarrow} \mathrm{HH}_{*}(A, A), c \otimes a \mapsto c \cap a,
$$

is a right action.

Following Tsygan's definition of a calculus, we want a left action. Therefore, we define as in [26], Definition 1.2,

$$
\ell^{*}(A, A) \otimes \ell_{*}(A, A) \rightarrow \ell_{*}(A, A), f \otimes c \mapsto i_{f}(c)=f \cdot c:=(-1)^{|c||f|} c \cap f .
$$

Explicitly

$$
i_{f}\left(m\left[a_{1}|\ldots| a_{n}\right]\right):=\sum_{p=0}^{n}(-1)^{|m||f|}\left(m \cdot f\left[a_{1}|\ldots| a_{p}\right]\right)\left[a_{p+1}|\ldots| a_{n}\right] .
$$

The sign in [8], (18), p. 82, is different. But with our choice of signs, we recover Proposition 2.6 in [8], p. 82. Indeed for $D, E \in \mathcal{C}^{*}(A, A)$ and $c \in \mathcal{C}_{*}(A, A)$,

$$
\begin{aligned}
D \cdot(E \cdot c) & =(-1)^{|c||E|} D \cdot(c \cap E) \\
& =(-1)^{|c||E|+|D||c|+|D||E|}(c \cap E) \cap D \\
& =(-1)^{|c||E|+|D||c|+|D||E|} c \cap(E \cup D) \\
& =(-1)^{|D||E|}(E \cup D) \cdot c .
\end{aligned}
$$

Since the cup product on $\mathrm{HH}^{*}(A, A)$ is graded commutative, for $D, E \in \mathrm{HH}^{*}(A, A)$ and $c \in \mathrm{HH}_{*}(A, A)$, we have

$$
D \cdot(E \cdot c)=(D \cup E) \cdot c,
$$

i.e., a left action. Note that in [33] we forgot to twist the right action by the sign $(-1)^{|c||f|}$ and so have also a sign problem.

Proposition 32 ([18], Theorem 3.4.3(ii)). Let $c \in \mathrm{HH}_{d}(A, A)$ such that the morphism of left $\mathrm{HH}^{*}(A, A)$-modules

$$
\mathrm{HH}^{p}(A, A) \stackrel{\cong}{\longrightarrow} \mathrm{HH}_{d-p}(A, A), \quad a \mapsto a \cdot c,
$$

is an isomorphism. If $B(c)=0$ then the Gerstenhaber algebra $\mathrm{HH}^{*}(A, A)$ equipped with $-B$ is a Batalin-Vilkovisky algebra.

Proof. Let us rewrite Victor Ginzburg's proof (or more precisely the proof we already gave in [33], Proposition 13 and Lemma 15) using explicitly Proposition 28 and our choice of signs. Denote by

$$
\mathrm{HH}^{p}(A, A) \otimes \mathrm{HH}_{j}(A, A) \rightarrow \mathrm{HH}_{j-p+1}(A, A), \quad a \otimes x \mapsto L_{a}(x),
$$


the action of the suspended graded Lie algebra $s \mathrm{HH}^{*}(A, A)$ on $\mathrm{HH}_{*}(A, A)$. Gelfand, Daletski and Tsygan [15] proved that the Gerstenhaber algebra $\operatorname{HH}^{*}(A, A)$ and Connes' boundary map $B$ on $\mathrm{HH}_{*}(A, A)$ form a calculus [8], p. 93. In particular, we have the two relations

$$
L_{a}=\left[B, i_{a}\right]
$$

and [8], Proposition 2.9, p. 83,

$$
i_{\{a, b\}}=(-1)^{|a|+1}\left[L_{a}, i_{b}\right] .
$$

Therefore

$$
i_{\{a, b\}}=(-1)^{|a|+1}\left[\left[B, i_{a}\right], i_{b}\right]=\left[\left[i_{a}, B\right], i_{b}\right] .
$$

The operator $\Delta$ on $\mathrm{HH}^{*}(A, A)$ is defined by

$$
(\Delta a) \cdot c:=-B(a \cdot c) \text { for any } a \in \mathrm{HH}^{*}(A, A) .
$$

Thus $B(c)=0$ implies $\Delta(1)=0$. Since we have a left action (eq. (31)), $l_{a}(b) \cdot c=$ $(a \cup b) \cdot c=a \cdot(b \cdot c)=i_{a}(b \cdot c)$ and so eq. (34) is equivalent to

$$
l_{\{a, b\}}=-\left[\left[l_{a}, \Delta\right], l_{b}\right] .
$$

Therefore, by Proposition $28, \mathrm{HH}^{*}(A, A)$ is a Batalin-Vilkovisky algebra.

Remark 35 (Signs). i) In [8], Example 4.6, p. 93, Tsygan writes that it follows from [8], 2.9, p. 83, that $i_{\{a, b\}}=\left[L_{a}, i_{b}\right]$. As Tsygan has kindly confirmed, there should be a sign in this formula: from [8], 2.9, p. 83, the correct equation with the signs is equation (33) above or equivalently $i_{\{a, b\}}=\left[i_{a}, L_{b}\right]([38],(0.1))$.

ii) In a calculus there is a third relation that we do not use in this paper:

$$
L_{a b}=L_{a} i_{b}+(-1)^{|a|} i_{a} L_{b} .
$$

Since $a b=(-1)^{|a||b|} b a$,

$$
L_{a b}=(-1)^{|a||b|} L_{b a}=(-1)^{|a||b|} L_{b} i_{a}+(-1)^{(|a|+1)|b|} i_{b} L_{a}
$$

and therefore

$$
\left[L_{a}, i_{b}\right]=(-1)^{|a||b|}\left[L_{b}, i_{a}\right] .
$$

Since $\{a, b\}=-(-1)^{(|a|+1)(|b|+1)}\{b, a\}$, if we suppose like in [8], Example 4.6, p. 93, that $i_{\{a, b\}}=\left[L_{a}, i_{b}\right]$, we obtain that

$$
\left[L_{a}, i_{b}\right]=-(-1)^{(|a|+1)(|b|+1)}\left[L_{b}, i_{a}\right] .
$$

The two equations (36) and (37) seem incoherent. Therefore the definition of calculus in [8], Definition 4.3, p. 33, has some sign problem.

On the contrary, if we suppose (33), we obtain again (36). 


\section{Proof of the main theorem for path-connected groups}

Cap products associated to coalgebras. Let $C$ be a (differential graded) coalgebra. Then its dual $C^{\vee}$ is a (differential graded) algebra. Let $N$ be a left $C$-comodule. Denote by $\Delta_{N}: N \rightarrow C \otimes N$ the structure map. Let $\cap: N \otimes C^{\vee} \rightarrow N$ be the composite

$$
N \otimes C^{\vee} \stackrel{\Delta_{N} \otimes C^{\vee}}{\longrightarrow} C \otimes N \otimes C^{\vee} \stackrel{C \otimes \tau}{\longrightarrow} C \otimes C^{\vee} \otimes N \stackrel{\text { ev } \otimes N}{\longrightarrow} \mathbb{k} \otimes N \cong N .
$$

Here $\tau$ denotes the twist map given by $n \otimes \varphi \mapsto(-1)^{|n||\varphi|} \varphi \otimes n$ and ev is the evaluation map defined by $\operatorname{ev}(c \otimes \varphi)=(-1)^{|\varphi||c|} \varphi(c)$. Then $N$ equipped with the cap product is a right $C^{\vee}$-module [37], Proposition 2.1.1. In this paper we are only interested in the case $N=C$.

Example 39. Let $X$ be any topological space. The (normalized or unnormalized) singular chains $S_{*}(X)$ of $X$ form a differential graded coalgebra [30], p. 244-45. The cap product $\cap: S_{*}(X) \otimes S^{*}(X) \rightarrow S_{*}(X)$ defined by (38) associated to $C=S_{*}(X)$ is the usual cap product.

Example 40. Let $A$ be any augmented differential graded algebra. Then the reduced (normalized or not) bar construction $B(A)=\mathcal{C}_{*}(A, \mathbb{k})$ is a differential graded coalgebra. The diagonal $\Delta: B(A) \rightarrow B(A) \otimes B(A)$ is given by

$$
\Delta\left(\left[a_{1}|\ldots| a_{n}\right]\right)=\sum_{p=0}^{n}\left[a_{1}|\ldots| a_{p}\right] \otimes\left[a_{p+1}|\ldots| a_{n}\right] .
$$

The cap product defined by (38) associated to $C=B(A)$ is given by

$$
\begin{gathered}
\cap: B(A) \otimes B(A)^{\vee} \rightarrow B(A), \\
{\left[a_{1}|\ldots| a_{n}\right] \cap f=\sum_{p=0}^{n}(-1)^{|f|\left(\left|a_{1}\right|+\cdots+\left|a_{n}\right|+n\right)} f\left(\left[a_{1}|\ldots| a_{p}\right]\right)\left[a_{p+1}|\ldots| a_{n}\right] .}
\end{gathered}
$$

Thus this cap product coincides with the cap product $\cap: \mathscr{\ell}_{*}(A, \mathbb{k}) \otimes \mathcal{C}^{*}(A, \mathbb{k}) \rightarrow$ $\mathcal{C}_{*}(A, \mathbb{k})$ on the Hochschild (co)chain complex defined by (8) in the case $N=B=\mathbb{k}$.

Proposition 41. Let $f: C \stackrel{\simeq}{\longrightarrow} D$ be a quasi-isomorphism of coalgebras. Suppose that $C$ and $D$ are $\mathbb{k}-$ free. Let $\tilde{c} \in C$ and $\tilde{d} \in D$ such that $\tilde{d}=H_{*}(f)([\tilde{c}])$. Consider the cap products defined by (38) associated to the coalgebras $C$ and $D$. Then the morphism of right $C^{\vee}$-modules $\tilde{c} \cap-: C^{\vee} \rightarrow C$ given by $a \mapsto \tilde{c} \cap$ a is a quasiisomorphism if and only if the morphism of right $D^{\vee}$-modules $\tilde{d} \cap-: D^{\vee} \rightarrow D$ given by $a \mapsto \tilde{d} \cap a$ is a quasi-isomorphism.

Proof. The transpose of $f: f^{\vee}: D^{\vee} \rightarrow C^{\vee}$ is a morphism of differential graded algebras. Therefore $f^{\vee}$ is a morphism of right $D^{\vee}$-modules. Dually, since $f$ is a 
morphism of coalgebras, $f$ is a morphism of left $D$-comodules and therefore $f$ is also a morphism of right $D^{\vee}$-modules by (38), i.e., $f\left(c \cap f^{\vee}(\varphi)\right)=f(c) \cap \varphi$ for any $c \in C$ and $\varphi \in D^{\vee}$. Note that if $f$ is the coalgebra map $S_{*}(\lambda): S_{*}(X) \rightarrow S_{*}(Y)$ induced by a continuous map $\lambda: X \rightarrow Y$, this formula is well known ([3], Chapter VI, Theorem 5.4, or [21], p. 241).

The composite of the morphisms of right $D^{\vee}$-modules

$$
D^{\vee} \stackrel{f^{\vee}}{\longrightarrow} C^{\vee} \stackrel{\tilde{c} \cap-}{\longrightarrow} C \stackrel{f}{\rightarrow} D
$$

maps 1 to $f(\tilde{c})$ and therefore coincides with the morphism of right $D^{\vee}$-modules $D^{\vee} \rightarrow D, a \mapsto f(\tilde{c}) \cap a$. Since $[\tilde{d}]=[f(\tilde{c})]$, the two maps $a \mapsto f(\tilde{c}) \cap a$ and $a \mapsto \tilde{d} \cap a$ coincide after passing to homology. Therefore after passing to homology the square

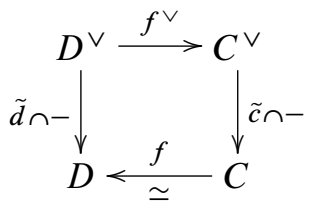

commutes. Since both $C$ and $D$ are $\mathbb{k}$-free and $\mathbb{k}$ is a principal ideal domain, by naturality of the universal coefficient theorem for cohomology, $H_{*}\left(f^{\vee}\right)$ is an isomorphism because $H_{*}(f)$ is an isomorphism. The proposition now follows from the square (42).

Theorem 43. Let $M$ be a simply-connected oriented Poincaré duality space offormal dimension $d$. Let $G$ be a topological group such that $M$ is a classifying space for $G$ or let $G$ be $\Omega M$ the (Moore) pointed loop space on $M$. Let $[M] \in H_{d}(M)$ be its fundamental class. Let $c$ the image of $[M]$ through the composite

$$
H_{*}(M) \stackrel{H_{*}(s)}{\longrightarrow} H_{*}(\mathrm{~L} M) \stackrel{\mathrm{BFG}^{-1}}{\longrightarrow} \mathrm{HH}_{*}\left(S_{*}(G), S_{*}(G)\right) .
$$

a) The morphism of left $\mathrm{HH}^{*}\left(S_{*}(G), S_{*}(G)\right)$-modules

$$
\mathbb{D}^{-1}: \mathrm{HH}^{p}\left(S_{*}(G), S_{*}(G)\right) \stackrel{\cong}{\longrightarrow} \mathrm{HH}_{d-p}\left(S_{*}(G), S_{*}(G)\right), \quad a \mapsto a \cdot c,
$$

is an isomorphism.

b) The Gerstenhaber algebra $\mathrm{HH}^{*}\left(S_{*}(G), S_{*}(G)\right)$ equipped with the operator $\Delta:=-\mathbb{D} \circ B \circ \mathbb{D}^{-1}$ is a Batalin-Vilkovisky algebra.

Here $s$ denotes $s: M \hookrightarrow \mathrm{L} M$ the inclusion of the constant loops into LM and BFG is the isomorphism of graded $\mathbb{k}$-modules between the free loop space homology of $M$ and the Hochschild homology of $S_{*}(G)$ introduced by Burghelea, Fiedorowicz [5] and Goodwillie [19]. Finally $B$ denotes Connes' boundary on $\mathrm{HH}_{*}\left(S_{*}(G), S_{*}(G)\right)$. 
Remark 44. We expect that the above theorem can be extended to any path-connected topological monoid $G$ instead of just the topological monoid of pointed Moore loop spaces $\Omega M$ or instead of just any topological group.

Proof. By [10], Proposition 6.13 in the case $F=p t$, when $G$ is a topological group or by [10], Theorem 6.3 , when $G=\Omega M$, there exists a differential graded coalgebra $B\left(S_{*}(E G) ; S_{*}(G) ; \mathbb{k}\right)$ and two quasi-isomorphisms of coalgebras

$$
B\left(S_{*}(G)\right) \stackrel{\simeq}{\longleftarrow} B\left(S_{*}(E G) ; S_{*}(G) ; \mathbb{k}\right) \stackrel{\simeq}{\longrightarrow} S_{*}(M) .
$$

The induced isomorphism in homology is the well-known isomorphism due to Moore [31], Corollary 7.29,

$$
\theta: \operatorname{Tor}^{S_{*}(G)}(\mathbb{k}, \mathbb{k})=H_{*}\left(B\left(S_{*}(G)\right)\right) \stackrel{\cong}{\cong} H_{*}(M) .
$$

Let $[m] \in H_{*}\left(B\left(S_{*}(G)\right)\right)$ such that $\theta([m])=[M]$. By Proposition 41 and Example 40 , the cap product with $[m],[m] \cap-: B\left(S_{*}(G)\right)^{\vee} \stackrel{\simeq}{\longrightarrow} B\left(S_{*}(G)\right), a \mapsto[m] \cap a$, is a quasi-isomorphism.

Denote by ev : LM $\rightarrow M, l \mapsto l(0)$, the evaluation map. The isomorphism BFG of Goodwillie, Burghelea and Fiedorowicz fits into the commutative square

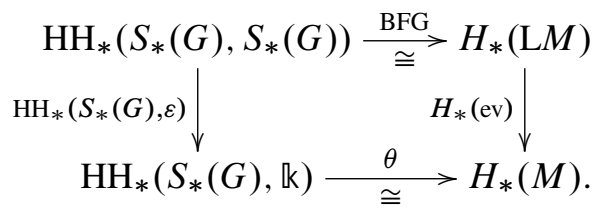

Here $\varepsilon$ denote the augmentation of $S_{*}(G)$. Let $c:=\mathrm{BFG}^{-1} \circ H_{d}(s)([M])$. Since $s$ is a section of the evaluation map ev, $\operatorname{HH}_{*}\left(S_{*}(G), \varepsilon\right)(c)=[m]$. So the hypotheses of Statement 9 are satisfied for $A=S_{*}(G)$.

Let $N$ be any non-negatively graded $S_{*}(G)$-bimodule. Since $M$ is simply connected, by Corollary 13, we obtain that the morphism

$$
\mathcal{D}^{-1}: \mathrm{HH}^{p}\left(S_{*}(G), N\right) \stackrel{\cong}{\longrightarrow} \mathrm{HH}_{d-p}\left(S_{*}(G), N\right), \quad a \mapsto c \cap a,
$$

is an isomorphism. By taking $N=S_{*}(G)$ and by passing from a right action to a left action, we obtain a) from (30).

The isomorphism BFG of Goodwillie, Burghelea and Fiedorowicz satisfies $\Delta \circ \mathrm{BFG}=\mathrm{BFG} \circ B$. Consider $M$ equipped with the trivial $S^{1}$-action. The section $s: M \hookrightarrow \mathrm{L} M$ is $S^{1}$-equivariant. Since

$$
B(c)=B \circ \mathrm{BFG}^{-1} \circ H_{d}(s)([M])=\mathrm{BFG}^{-1} \circ \Delta \circ H_{d}(s)([M])=0,
$$

by Proposition 32, we obtain b). 


\section{Proof of the main theorem for discrete groups}

Theorem 45. Let $G$ be a discrete group such that its classifying space $K(G, 1)$ is an oriented Poincaré duality space of formal dimension $d$. Let $[M] \in H_{d}(G, \mathbb{k})$ be a fundamental class. Let $c$ be the image of $[M]$ by $\operatorname{Tor}_{*}^{E}(\eta, \eta): H_{*}(G, \mathbb{k}) \rightarrow$ $\mathrm{HH}_{*}(\mathrm{k}[G], \mathrm{k}[G])$ (Property 19ii)).

a) The morphism of left $\mathrm{HH}^{*}(\mathbb{k}[G], \mathbb{k}[G])$-modules

$$
\mathbb{D}^{-1}: \mathrm{HH}^{p}(\mathbb{k}[G], \mathbb{k}[G]) \stackrel{\cong}{\longrightarrow} \mathrm{HH}_{d-p}(\mathbb{k}[G], \mathbb{k}[G]), \quad a \mapsto a \cdot c,
$$

is an isomorphism.

b) The Gerstenhaber algebra $\mathrm{HH}^{*}(\mathbb{k}[G], \mathbb{k}[G])$ equipped with the operator $\Delta:=$ $-\mathbb{D} \circ B \circ \mathbb{D}^{-1}$ is a Batalin-Vilkovisky algebra.

Proof. Let $N$ be any ungraded $\mathbb{k}[G]$-bimodule. Since, by hypothesis, $G$ is an orientable Poincaré duality group, the cap product with $[M]$ in group (co)homology gives an isomorphism ([4], $10.1 \mathrm{iv),} \mathrm{Remark} 1$ and Example 1, p. 222, [16], Theorem 15.3.1)

$$
[M] \cap-: H^{p}(G, \tilde{N}) \stackrel{\cong}{\longrightarrow} H_{d-p}(G, \tilde{N}), a \mapsto[M] \cap a .
$$

Therefore, by Corollary 20, the cap product with $c=\sigma([M])$ in Hochschild (co)homology gives the isomorphism

$$
c \cap-: \mathrm{HH}^{p}(\mathbb{k}[G], N) \rightarrow \mathrm{HH}_{d-p}(\mathbb{k}[G], N), \quad a \mapsto c \cap a .
$$

Taking $N=\mathbb{k}[G]$ and passing from a right action to left action as in (30), we obtain a).

By i) of Property $19, \sigma: H_{*}(G ; \mathbb{k}) \rightarrow \mathrm{HH}_{*}(\mathbb{k}[G], \mathbb{k}[G])$ commutes with Connes' boundary map $B$ on $H_{*}(G ; \mathbb{k})$ and on $\mathrm{HH}_{*}(\mathbb{k}[G], \mathbb{k}[G])$. By a well-known result of Karoubi (see, e.g., [28], E.7.4.8, or [40], Theorem 9.7.1), Connes' boundary map $B$ is trivial on $H_{*}(G ; \mathbb{k})$. Therefore $B(c)=B \circ \sigma([M])=\sigma \circ B([M])=0$. By applying Proposition 32, we obtain b).

Property 46. Let $A$ and $B$ be two algebras (differential graded if we want). Let $N$ be an $(A, A \otimes B)$-bimodule. Let $c \in \mathrm{HH}_{d}(A, A)$. Then

i) $\mathrm{HH}^{*}(A, N)$ and $\mathrm{HH}_{*}(A, N)$ are two right $B$-modules, and

ii) the cap product

$$
c \cap-: \mathrm{HH}^{p}(A, N) \rightarrow \mathrm{HH}_{d-p}(A, N), \quad a \mapsto c \cap a,
$$

is a morphism of right $B$-modules.

Proof. Since $N$ is an $\left(A^{e}, B\right)$-bimodule, $\mathcal{C}^{*}(A, N) \cong \operatorname{Hom}_{A^{e}}(B(A ; A ; A), N)$ is a (differential graded) right $B$-module and its homology $\mathrm{HH}^{*}(A, N)$ is a right $B$ module. Similarly $\ell_{*}(A, N) \cong N \otimes_{A^{e}} B(A ; A ; A)$ and $\mathrm{HH}_{*}(A, N)$ are two right 
$B$-modules. Let $c$ be $a\left[a_{1}|\ldots| a_{n}\right] \in \mathcal{C}_{n}(A, A)$. Let $f \in \mathcal{C}^{p}(A, N)$. By definition, $c \cap f:= \pm a f\left(\left[a_{1}|\ldots| a_{p}\right]\right)\left[a_{p+1}|\ldots| a_{n}\right]$. Therefore

$$
\begin{aligned}
(c \cap f) \cdot b & = \pm a f\left(\left[a_{1}|\ldots| a_{p}\right]\right) b\left[a_{p+1}|\ldots| a_{n}\right] \\
& = \pm a(f \cdot b)\left(\left[a_{1}|\ldots| a_{p}\right]\right)\left[a_{p+1}|\ldots| a_{n}\right]=c \cap(f \cdot b)
\end{aligned}
$$

for any $b \in B$.

Remark 47. We will be only interested in the case $N=A \otimes A$ and $B=A^{e}$. Here the $A$-bimodule structure on $N$ is given by $a \cdot(x \otimes y) \cdot b=a x \otimes y b$ and is called the outer structure [18], (1.5.1). And the right $B$-module on $N$ is given by $(x \otimes y) \cdot(a \otimes b)=x a \otimes b y, x \otimes y \in N, a \otimes b \in B$ and is called the inner structure.

Definition 48 ([18], Definition 3.2.3, formula (3.2.5), Remark 3.2.8, or simply [2], Definition 2.1)). An ungraded algebra $A$ is Calabi-Yau of dimension $d$ if

i) viewed as an $A$-bimodule over itself, $A$ admits a finite resolution by finite type projective $A$-bimodules, i.e., there exists an exact sequence of $A^{e}$-projective finite type module of the form

$$
0 \rightarrow P_{i} \rightarrow P_{i-1} \rightarrow \cdots \rightarrow P_{1} \rightarrow P_{0} \rightarrow A \rightarrow 0
$$

ii) $\mathrm{HH}^{k}(A, A \otimes A)=0$ for all $k \neq d$, and

iii) as $(A, A)$-bimodule, $\mathrm{HH}^{d}(A, A \otimes A)$ is isomorphic to $A$. (Here the $(A, A)$ bimodule on $\mathrm{HH}^{*}(A, A \otimes A)$ is given by Property 46 and Remark 47.)

Proposition 49 ([18], Remark 3.4.2, stated without proof). Let $A$ be an ungraded algebra, and let $c \in \mathrm{HH}_{d}(A, A)$. Suppose that, for every A-bimodule $N$, $c \cap-: \mathrm{HH}^{p}(A, N) \stackrel{\cong}{\rightrightarrows} \mathrm{HH}_{d-p}(A, N), a \mapsto c \cap a$, is an isomorphism. Then $A$ satisfies conditions ii) and iii) of Definition 48.

Proof. Let $N$ be a free $(A, A)$-bimodule. Then $\mathrm{HH}_{*}(A, N)=0$ if $* \neq 0$. Therefore $\mathrm{HH}^{k}(A, N)=0$ if $k \neq d$. Suppose moreover that $N$ is a $(A, A \otimes B)$-bimodule. The quasi-isomorphism of complexes $\ell_{*}(A, N) \cong N \otimes_{A^{e}} B(A ; A ; A) \stackrel{\simeq}{\longrightarrow} N \otimes_{A^{e}} A$ is a morphism of right $B$-modules. By Property 46 ,

$$
c \cap-: \mathrm{HH}^{d}(A, N) \rightarrow \mathrm{HH}_{0}(A, N) \cong N \otimes_{A^{e}} A
$$

is an isomorphism of right $B$-modules.

Let $N$ be the $(A, A)$-bimodule $A \otimes A$ with the outer structure and $B=A^{e}$ (see Remark 47). Then $N \otimes_{A^{e}} A=(A \otimes A) \otimes_{A^{e}} A \stackrel{\cong}{\cong},(x \otimes y) \otimes_{A^{e}} m \mapsto y m x$, is an isomorphism whose inverse is the map mapping $a \mapsto(1 \otimes 1) \otimes_{A^{e}} a$. A straightforward calculation shows that these isomorphisms are right $A^{e}$-linear. Therefore, we have proved that $\mathrm{HH}^{d}(A, A \otimes A)$ is isomorphic to $A$ as right $A^{e}$-module. 
Theorem 50. Let $\mathbb{k}$ be any commutative ring. Let $G$ be an orientable Poincaré duality group of dimension $d$. Then its group ring $\mathbb{k}[G]$ is a Calabi-Yau algebra of dimension $d$.

When $\mathbb{k}$ is a field of characteristic 0 or of characteristic prime to the cardinal of $G$, this theorem was proved by Kontsevich [18], Corollary 6.1.4, and Lambre [26], Lemme 6.2.

Proof. By [4], Remark 2, p. 222, there exists a finite resolution $P \stackrel{\simeq}{\longrightarrow} \mathbb{k}$ of $\mathbb{k}$ by finite type projective $\mathbb{k}[G]$-left modules. Then $X:=\mathbb{k}\left[G \times G^{\mathrm{op}}\right] \otimes_{\mathbb{k}[G]} P \stackrel{\simeq}{\longrightarrow}[G]$ is a finite type resolution of $\mathbb{k}[G]$ by finite type projective $\mathbb{k}[G]$-bimodules.

In the course of the proof of Theorem 45, we saw that, for any $\mathbb{k}[G]$-bimodule $N, c \cap-: \mathrm{HH}^{p}(\mathbb{k}[G], N) \stackrel{\cong}{\longrightarrow} \mathrm{HH}_{d-p}(\mathbb{k}[G], N), a \mapsto c \cap a$, is an isomorphism. Therefore, by Proposition 49, $\mathbb{k}[G]$ is a Calabi-Yau algebra of dimension $d$.

\section{String topology of classifying spaces}

In [7], Chataur and the author, and in [1], Behrend, Ginot, Noohi and Xu developed a string topology theory dual to Chas-Sullivan string topology.

Theorem 51 ([1], [7]). Let $G$ be a path-connected compact Lie group of dimension $d$. Denote by BG its classifying space. Then the shifted free loop space cohomology $H^{*+d}(\mathrm{LBG})$ is a (possibly non-unital) Batalin-Vilkovisky algebra.

The goal of this section is to prove the following theorem:

Theorem 52. Let $G$ be a path-connected compact Lie group of dimension d. Denote by $S^{*}(B G)$ the singular cochains on the classifying space of $G$.

a) There exists an explicit isomorphism of left $\mathrm{HH}^{*}\left(S^{*}(B G), S^{*}(B G)\right)$-modules

$$
\mathbb{D}^{-1}: \mathrm{HH}^{p}\left(S^{*}(B G), S^{*}(B G)\right) \stackrel{\cong}{\rightrightarrows} \mathrm{HH}_{-d-p}\left(S^{*}(B G), S^{*}(B G)\right) .
$$

b) The Gerstenhaber algebra $\mathrm{HH}^{*}\left(S^{*}(B G), S^{*}(B G)\right)$ equipped with the operator $\Delta:=-\mathbb{D} \circ B \circ \mathbb{D}^{-1}$ is a Batalin-Vilkovisky algebra.

Both Batalin-Vilkovisky algebras in Theorems 51 and 52 are determined by an orientation class of $H_{d}(G)$. In [23], Jones gave an isomorphism of graded vector spaces

$$
J: \mathrm{HH}_{*}\left(S^{*}(B G), S^{*}(B G)\right) \stackrel{\cong}{\longrightarrow} H^{*}(\mathrm{~L} B G) .
$$

We guess that the isomorphism $J \circ \mathbb{D}^{-1}: \operatorname{HH}^{*}\left(S^{*}(B G), S^{*}(B G)\right) \stackrel{\cong}{\longrightarrow} H^{*+d}(\mathrm{~L} B G)$ of graded vector spaces is a morphism of Batalin-Vilkovisky algebras.

Theorem 52 is the Eckmann-Hilton or Koszul dual of the following theorem proved by Chataur and the author. 
Theorem 53 ([7], Theorem 54). Let $G$ be a path-connected compact Lie group of dimensiond. Denote by $S_{*}(G)$ the algebra of singular chains of $G$. Consider Connes' coboundary map $H\left(B^{\vee}\right)$ on the Hochschild cohomology of $S_{*}(G)$ with coefficients in its dual $\mathrm{HH}^{*}\left(S_{*}(G) ; S^{*}(G)\right)$. Then there is an isomorphism

$$
\mathcal{D}^{-1}: \mathrm{HH}^{p}\left(S_{*}(G) ; S_{*}(G)\right) \stackrel{\cong}{\longrightarrow} \mathrm{HH}^{p+d}\left(S_{*}(G) ; S^{*}(G)\right)
$$

of graded vector spaces of upper degree $d$ such that the Gerstenhaber algebra $\mathrm{HH}^{*}\left(S_{*}(G) ; S_{*}(G)\right)$ equipped with the operator $\Delta=\mathscr{D} \circ H\left(B^{\vee}\right) \circ D^{-1}$ is a Batalin-Vilkovisky algebra.

\subsection{Frobenius algebras}

Definition 54. Let $A$ be a differential graded algebra. We say that $A$ is a Frobenius algebra if there is a quasi-isomorphism of right $A$-modules $A \stackrel{\simeq}{\longrightarrow} A^{\vee}$. In particular, a graded algebra $A$ is a Frobenius algebra if $A$ is isomorphic as right $A$-modules to its dual $A^{\vee}$.

Property 55 ([29], Theorem 9.8). Let $A$ be a differential graded algebra. Then $A$ is a Frobenius algebra if and only if its homology $H(A)$ is a Frobenius algebra.

Proof. Let $M$ be any left $A$-module. A straightforward computation shows that the linear map $\mu: H(\operatorname{Hom}(M, \mathbb{k})) \rightarrow \operatorname{Hom}(H(M), \mathbb{k})$ mapping a cycle $f: M \rightarrow \mathbb{k}$ to $H(f): H(M) \rightarrow \mathbb{k}$ is a morphism of right $H(A)$-modules. Since in this section $\mathbb{k}$ is a field, by the universal coefficient theorem for cohomology, this map $\mu$ is an isomorphism. We are only interested in the case $M=A$.

Suppose that we have an quasi-isomorphism of right $A$-modules $\Theta: A \stackrel{\simeq}{\longrightarrow} A^{\vee}$. Then the composite $H(A) \stackrel{H(\Theta)}{\longrightarrow} H\left(A^{\vee}\right) \stackrel{\mu}{\rightarrow} H(A)^{\vee}$ is an isomorphism of right $H(A)$-modules.

Conversely, suppose that we have an isomorphism $\Theta: H(A) \stackrel{\cong}{\longrightarrow} H(A)^{\vee}$ of right $H(A)$-modules. Then the composite $H(A) \stackrel{\Theta}{\rightarrow} H(A)^{\vee} \stackrel{\mu^{-1}}{\rightarrow} H\left(A^{\vee}\right)$ is also an isomorphism of right $H(A)$-modules. Let $x$ be a cycle of $A^{\vee}$ such that $\mu^{-1} \circ \Theta(1)=$ [x].The morphism of right $A$-modules $A \rightarrow A^{\vee}, a \mapsto x a$, coincides in homology with the isomorphism $\mu^{-1} \circ \Theta$.

Corollary 56. Let $A$ and $B$ be two differential graded algebras such that $H(A) \cong$ $H(B)$ as graded algebras. Then $A$ is Frobenius if and only if $B$ is.

Observe that there does not necessarily exist a quasi-isomorphism of algebras $f: A \stackrel{\simeq}{\longrightarrow}$. (Compare with Proposition 41 or [29], Corollary 9.9.)

Property 57. Let $A$ be a graded algebra and let $C$ be a graded coalgebra. Consider a bilinear form $\langle\rangle:, C \otimes A: \rightarrow \mathbb{k}$. Let $\phi: A \rightarrow C^{\vee}, a \mapsto\langle-, a\rangle$, and let $\psi: C \rightarrow A^{\vee}, c \mapsto\langle c,-\rangle$, be the right and left adjoints. Suppose that $\phi$ is a morphism of graded algebras. Then 
i) $\psi$ is a morphism of right $A$-modules with respect to the cap product (38) associated to the coalgebra $C$, i.e., $\psi(c \cap \phi(a))=\psi(c) \cdot a$ for any $c \in C$ and $a \in A$,

ii) if $A$ is non-negatively graded and of finite type in each degree then $\psi: C \rightarrow A^{\vee}$ is a morphism of graded coalgebras.

Proof. i) Let $\Delta c=\sum c^{\prime} \otimes c^{\prime \prime}$ be the diagonal of $c$. By definition, the cap product $c \cap \phi(a)$ is equal to $\sum(-1)^{|c||| a \mid}\left\langle c^{\prime}, a\right\rangle c^{\prime \prime}$. Therefore $\psi(c \cap \phi(a))$ is the form on $A$ mapping $x \in A$ to $\sum(-1)^{|c "||a|}\left\langle c^{\prime}, a\right\rangle\left\langle c^{\prime \prime}, x\right\rangle$. On the other hand, $\psi(c) \cdot a$ is the form on $A$ mapping $x \in A$ to $\langle c, a x\rangle$. But $\phi$ is a morphism of algebras if only and if $\langle c, a x\rangle=\sum(-1)^{|c||| a \mid}\left\langle c^{\prime}, a\right\rangle\left\langle c^{\prime \prime}, x\right\rangle$ for every $a, x \in A$ and $c \in C$.

Let us give a well-known application of i) of Property 57. Let $C=S_{*}(M)$ and $A=C^{\vee}=S^{*}(M)$. We obtain that the quasi-isomorphism $\psi: S_{*}(M) \rightarrow S^{*}(M)^{\vee}$ is a morphism of $S^{*}(M)$-modules [13], Section 7. Therefore, by Poincaré duality, $S^{*}(M)$ is a Frobenius algebra, and so is $H^{*}(M)$.

9.2. String topology of manifolds. Let $M$ be a closed oriented $d$-dimensional smooth manifold. Denote by $\mathbb{H}_{*}(M):=H_{*+d}(M)$. Poincaré duality [21], Theorem 3.30, gives an isomorphism of graded algebras

$$
H^{*}(M) \cong \mathbb{H}_{*}(M),
$$

where

- the product on $H^{*}(M)$ is the cup product $H^{*}(\Delta)$,

- the product on $\mathbb{H}_{*}(M)$ is the intersection product $\Delta_{!}$, and

- the fundamental class $[M] \in H_{d}(M)$ is the unit of $\mathbb{H}_{*}(M)$.

Chas and Sullivan have defined a Batalin-Vilkovisky algebra on $\mathbb{H}_{*}(\mathrm{~L} M):=$ $H_{*+d}(\mathrm{~L} M)$. The Chas-Sullivan loop product on $\mathbb{H}_{*}(\mathrm{~L} M)$ mixes the intersection

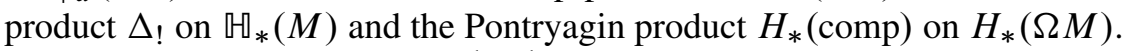

More precisely, let $\tilde{\Delta}: M^{S^{1} \vee S^{1}} \hookrightarrow \mathrm{L} M \times \mathrm{L} M$ be the inclusion map and let comp: $M^{S^{1} \vee S^{1}} \rightarrow \mathrm{L} M$ be the map obtained by composing loops. The ChasSullivan loop product is the composite

$$
H_{*}(\mathrm{~L} M \times \mathrm{L} M) \stackrel{\tilde{\Delta}_{!}}{\longrightarrow} H_{*-d}\left(M^{S^{1} \vee S^{1}}\right) \stackrel{H_{*} \text { (comp) }}{\longrightarrow} H_{*-d}(\mathrm{~L} M) .
$$

The loop product admits $H_{d}(s)([M])$ as unit. More generally $H_{*}(s): \mathbb{H}_{*}(M) \rightarrow$ $\mathbb{W}_{*}(\mathrm{~L} M)$ is a morphism of algebras preserving the units. Let $i: \Omega M \hookrightarrow \mathrm{L} M$ be the inclusion of the pointed loops into the free loops. The shriek map of $i$, called the intersection map, $i_{!}: \mathbb{H}_{*}(\mathrm{~L} M) \rightarrow H_{*}(\Omega M)$, is also a morphism of algebras preserving the units [6], Proposition 3.4.

The unit of the Batalin-Vilkovisky algebra $\mathbb{H}_{*}(\mathrm{~L} M)$ and the fact that $\Delta 1=0$ in any unital Batalin-Vilkovisky algebras was the key for proving Theorem 43. 
9.3. Versus string topology of classifying spaces. Let $G$ be a path-connected Lie group of dimension $d$. Denote $\mathbb{H}^{*}(\Omega B G)=H^{*+d}(\Omega B G)$. Since $H_{*}(\Omega B G)$ is a finite dimensional Hopf algebra, $H_{*}(\Omega B G)$ is a Frobenius algebra: there is an isomorphism of right $H_{*}(\Omega B G)$-modules [7], Section 4.1,

$$
\Theta: H_{*}(\Omega B G) \cong \mathbb{Q}^{*}(\Omega B G) .
$$

By [37], Theorem 5.1.2 (with left Hopf modules instead of right Hopf modules), the composite $H_{*}(\Omega B G) \stackrel{S}{\rightarrow} H_{*}(\Omega B G) \stackrel{\Theta}{\rightarrow} H^{*}(\Omega B G)$ of the antipode of the Hopf algebra $H_{*}(\Omega B G)$ and of $\Theta$ is an isomorphism of left Hopf modules over $H_{*}(\Omega B G)$, and so coincides with Poincaré duality.

Therefore this isomorphism $\Theta$ is an isomorphism of algebras if

- the product on $H_{*}(\Omega B G)$ is the Pontryagin product $H_{*}$ (comp),

- the product on $\mathbb{H}^{*}(\Omega B G)$ is the composite

$$
H^{*}(\Omega B G) \otimes H^{*}(\Omega B G) \stackrel{\tau}{\rightarrow} H^{*}(\Omega B G) \otimes H^{*}(\Omega B G) \stackrel{\text { comp }^{!}}{\longrightarrow} H^{*-d}(\Omega B G),
$$

where $\tau$ denotes the twist map given by $a \otimes b \mapsto(-1)^{|a||b|} b \otimes a$ and comp! is the shriek map of comp.

Of course, $\Theta(1)$ is the unit of the algebra $\mathbb{Q}^{*}(\Omega B G)$.

The product on $\mathbb{Q}^{*}(\mathrm{~L} B G):=H^{*+d}(\mathrm{~L} B G)$ mixes the cup product $H^{*}(\Delta)$ on $H^{*}(B G)$ and the product comp! on $\mathbb{H}^{*}(\Omega B G)$. More precisely, the product on $\mathbb{Q}^{*}(\mathrm{LBG})$ is the composite

$$
H^{*}(\mathrm{~L} B G \times \mathrm{L} B G) \stackrel{H^{*}(\tilde{\Delta})}{\longrightarrow} H^{*}\left(B G^{S^{1} \vee S^{1}}\right) \stackrel{\text { comp }^{!}}{\longrightarrow} H^{*-d}(\mathrm{~L} B G) .
$$

Comparing with the definition of the Chas-Sullivan loop product defined above, we see a general principle. In order to pass from string topology of manifolds to string topology of classifying spaces, you replace

- homology by cohomology,

- shriek map in homology like $\tilde{\Delta}_{\text {! }}$ by the map induced in singular cohomology like $H^{*}(\tilde{\Delta})$,

- maps induced in singular homology like $H_{*}$ (comp) by shriek map in cohomology like comp!

In particular, you never change the direction of arrows.

Guided by this general principle, we now transpose the proof of Theorem 43 into a proof of Theorem 52. Using this general principle, the product on $\mathbb{Q}^{*}(\mathrm{~L} B G)$ should have $s^{!}(1)$ as a unit. More generally $s^{!}: H^{*}(B G) \rightarrow \mathbb{Q}^{*}(\mathrm{~L} B G)$ should be a morphism of algebras preserving the units. Also $H^{*}(i): \mathbb{U}^{*}(\mathrm{~L} B G) \rightarrow \mathbb{U}^{*}(\Omega B G)$ should be a morphism of algebras preserving the units. The problem is that $s^{!}$is not easy to define [7], Remark 56, and that we have not yet proved the previous assertions. Instead, we are going only to prove the following lemma. 
Lemma 58. There exists an explicit element $\mathbb{I} \in H^{d}(\mathrm{LBG})$ such that $\Delta \mathbb{I}=0$ and such that the morphism $\Theta: H_{p}(\Omega B G) \stackrel{\cong}{\cong} H^{d-p}(\Omega B G), a \mapsto H^{d}(i)(\mathbb{I}) \cdot a$, of right $H_{*}(\Omega B G)$-modules is an isomorphism.

As explained above, we believe that $\mathbb{I}$ is the unit of the Batalin-Vilkovisky algebra $\mathrm{H}^{*}(\mathrm{~L} B G)$.

Proof. Let $\eta:\{e\} \rightarrow G$ be the unit of $G$. Consider $\eta_{!}: H_{d}(G) \rightarrow \mathbb{k}$ the shriek map of $\eta$. By Lemma 55 of [7], the morphism $H_{p}(G) \stackrel{\cong}{\cong} H^{d-p}(G), a \mapsto \eta ! \cdot a$, of right $H_{*}(G)$-modules is an isomorphism. Consider the commutative diagram of graded algebras

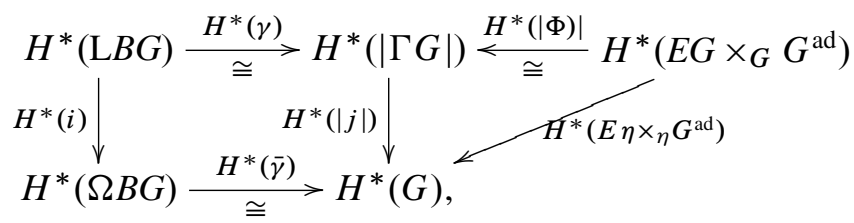

where the right triangle is the triangle considered in the proof of Theorem 54 of [7] and the left square is induced by the commutative square of topological spaces

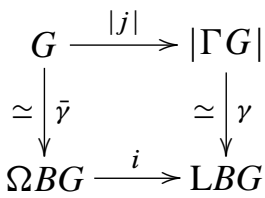

considered in the proof of Theorem 7.3.11 of [28]. Consider the equivariant Gysin map: $E G \times_{G} \eta^{!}: H^{*}(B G) \rightarrow H^{*+d}\left(E G \times_{G} G^{\text {ad }}\right)$. Let II be the image of 1 by the composite $H^{*}(\gamma)^{-1} \circ H^{*}(|\Phi|) \circ E G \times_{G} \eta^{!}$. In [7, (58)], we saw that $\Delta \mathbb{I}=0$. By Lemma 57 of [7], $H^{*}\left(E \eta \times_{\eta} G^{\text {ad }}\right)$ maps $E G \times_{G} \eta^{!}(1)$ to $\eta ! \in H_{d}(G)^{\vee}$. Therefore using the above commutative diagram, $H^{*}(i)(\mathbb{I})=H^{*}(\bar{\gamma})^{-1}(\eta !)$.

By Lemma 7.3.12 of [28], $\bar{\gamma}: G \stackrel{\simeq}{\longrightarrow} \Omega B G$ is the classical homotopy equivalence, which is well known to be a morphism of $H$-spaces. Therefore the isomorphism induced in homology, $H_{*}(\bar{\gamma}): H_{*}(G) \stackrel{\cong}{\cong} H_{*}(\Omega B G)$, is a morphism of algebras. Since $H_{*}(G)$ is a Frobenius algebra, $H_{*}(\Omega B G)$ is also a Frobenius algebra. More precisely, the morphism $\Theta: H_{p}(\Omega B G) \rightarrow H_{d-p}(\Omega B G)^{\vee}, a \mapsto H^{*}(\bar{\gamma})^{-1}\left(\eta_{!}\right) \cdot a$, of right $H_{*}(\Omega B G)$-modules is an isomorphism.

To finish the proof of Theorem 52, we need also the following algebraic results.

9.4. Bar and cobar construction. Let $C$ be a coaugmented DGC. Denote by $\bar{C}$ the kernel of the counit. The normalized cobar construction on $C$, denoted $\Omega C$, is the 
augmented differential graded algebra $\left(T\left(s^{-1} \bar{C}\right), d_{1}+d_{2}\right)$ where $d_{1}$ and $d_{2}$ are the unique derivations determined by

$$
d_{1} s^{-1} c=-s^{-1} d c \quad \text { and } \quad d_{2} s^{-1} c=\sum_{i}(-1)^{\left|x_{i}\right|} s^{-1} x_{i} \otimes s^{-1} y_{i}, \quad c \in \bar{C},
$$

where the reduced diagonal $\bar{\Delta} c=\sum_{i} x_{i} \otimes y_{i}$. We follow the sign convention of [9].

Remark 59 ([20], (A.6)). A bilinear form $\langle\rangle:, V \otimes W \rightarrow \mathbb{k}$ of graded vector spaces extends a bilinear form $\langle\rangle:, T V \otimes T W \rightarrow \mathbb{k}$ defined by

$$
\left\langle v_{1} \otimes \cdots \otimes v_{i}, w_{1} \otimes \cdots \otimes w_{i}\right\rangle= \pm \prod_{j=1}^{i}\left\langle v_{j}, w_{j}\right\rangle
$$

and $\left\langle v_{1} \otimes \cdots \otimes v_{i}, w_{1} \otimes \cdots \otimes w_{j}\right\rangle=0$ if $i \neq j$. Here again \pm is the sign given by the Koszul sign convention.

Proposition 60. Let $C$ be a coaugmented differential graded coalgebra. Denote by $A:=C^{\vee}$ the differential graded algebra dual of $C$. Let $\langle\rangle:, \mathrm{s} A \otimes s^{-1} C \rightarrow \mathbb{k}$ be the non-degenerate bilinear form defined by $\left\langle s a, s^{-1} c\right\rangle=(-1)^{|a|+1} a(c)$ in [20], p. 276 in the case $V=s^{-1} C$ and $X=A$. Consider the bilinearform $\langle\rangle:, B A \otimes \Omega C \rightarrow \mathbb{k}$ extending $\langle\rangle:, \mathrm{s} A \otimes s^{-1} C \rightarrow \mathbb{k}$ (Remark 59). Then

i) the right adjoint $\phi: \Omega C \rightarrow(B A)^{\vee}$ is a natural morphism of differential graded algebras and the left adjoint $\psi: B A \rightarrow(\Omega C)^{\vee}$ is a natural morphism of complexes,

ii) if $C$ is of finite type in each degree and $C=\mathbb{k} \oplus C_{\geq 2}$ then both $\phi$ and $\psi$ are isomorphisms,

iii) if $H(C)$ is of finite type in each degree and $C=\mathbb{k} \oplus C_{\geq 2}$ then both $H(\phi)$ and $H(\psi)$ are isomorphisms.

Proof. i) and ii) Denote by TA $W$ the tensor algebra on $W$, and by TC $V$ the tensor coalgebra on $V$ [20], p. 277-78. It is easy to check that the right adjoint map $\phi: \mathrm{TA} W \rightarrow \mathrm{TC} V^{\vee}$ of the bilinear map defined by Remark 59 is a morphism of graded algebras. In [32], proof of Theorem $6.1 \mathrm{ii}$ ), we have checked carefully that $\psi: \ell_{*}(A, A) \rightarrow(C \otimes \Omega C, \delta)^{\vee}$, where $(C \otimes \Omega C, \delta)$ is the cyclic cobar complex of $C$, is a morphism of complexes and an isomorphism if $C$ is of finite type in each degree and $\bar{C}=\bar{C}_{\geq 2}$. The same proof applies to $\psi: B A \rightarrow \Omega C^{\vee}$ as well.

iii) By Proposition 4.2 of [9], there exist a differential graded algebra of the form $(T V, d)$, where $V=V^{\geq 2}$ is of finite type in each degree, and a quasi-isomorphism $f: T V \stackrel{\simeq}{\longrightarrow} C^{\vee}$ of augmented differential graded algebras. By ii) of Property 57, the adjoint map $g: C \stackrel{\simeq}{\longrightarrow}\left(C^{\vee}\right)^{\vee} \stackrel{f^{\vee}}{\longrightarrow} T V^{\vee}$ is a quasi-isomorphism of coaugmented differential graded coalgebras [12], p. 56. Denote $D:=T V^{\vee}$. 
Since $\bar{C}_{\leq 1}=\bar{D}_{\leq 1}=0$, by Remark 2.3 of [9], $\Omega f: \Omega C \stackrel{\simeq}{\longrightarrow} \Omega D$ is a quasi-isomorphism of augmented differential graded algebras. Since $\mathbb{k}$ is a field, $f^{\vee}: D^{\vee} \stackrel{\simeq}{\longrightarrow} C^{\vee}$ is also a quasi-isomorphism of augmented differential graded algebras. By naturality of $\psi$, we have the commutative square of complexes

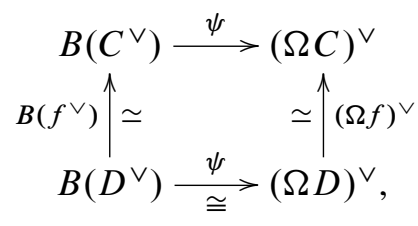

where the two vertical morphisms are quasi-isomorphisms. By ii) we have that $\psi: B\left(D^{\vee}\right) \stackrel{\cong}{\longrightarrow}(\Omega D)^{\vee}$ is an isomorphism. Therefore $\psi: B\left(C^{\vee}\right) \stackrel{\simeq}{\longrightarrow}(\Omega C)^{\vee}$ is a quasi-isomorphism. Similarly, one proves that $\phi: \Omega C \stackrel{\simeq}{\longrightarrow}\left(C^{\vee}\right)^{\vee}$ is a quasiisomorphism as well.

Proof of Theorem 52. The Eilenberg Moore formula gives an isomorphism of graded algebras $\mathcal{E M M}_{\mathcal{M}}: H_{*}(\Omega B G) \stackrel{\cong}{\longrightarrow} H\left(\Omega S_{*}(B G)\right)$. It follows from Proposition 60 iii) that $\psi: B S^{*}(B G) \stackrel{\simeq}{\longrightarrow} \Omega S_{*}(B G)^{\vee}$ is a quasi-isomorphism of complexes. The Jones isomorphism $J$ fits into the commutative diagram

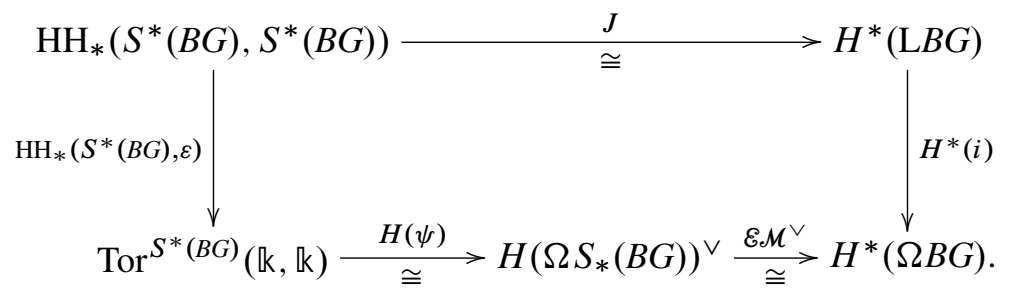

Consider the element $\mathbb{I} \in H^{d}(\mathrm{~L} B G)$ given by Lemma 58 . Let $c$ be $J^{-1}(\mathbb{I}) \in$ $\mathrm{HH}_{-d}\left(S^{*}(B G), S^{*}(B G)\right)$. Denote by $m \in B S^{*}(B G)$ a cycle such that its class $[m]$ is equal to $\mathrm{HH}_{-d}\left(S^{*}(B G), \varepsilon\right)(c)$.

Since $H_{*}(\Omega B G)$ is a Frobenius algebra, $H\left(\Omega S_{*}(B G)\right)$ is also a Frobenius algebra. More precisely, by Lemma 58, the morphism of right $H_{*}(\Omega B G)$-modules $H_{p}(\Omega B G) \stackrel{\cong}{\longrightarrow} H_{d-p}(\Omega B G)^{\vee}$ mapping 1 to $H^{d}(i)(\mathbb{I})$ is an isomorphism. Therefore the morphism $H_{p}\left(\Omega S_{*}(B G)\right) \stackrel{\cong}{\longrightarrow} H_{d-p}\left(\Omega S_{*}(B G)\right)^{\vee}$ of right $H\left(\Omega S_{*}(B G)\right)$ modules mapping 1 to $\left(\mathcal{E} \mathcal{M}^{\vee}\right)^{-1} \circ H^{d}(i)(\mathbb{I})$ is an isomorphism. Since the above diagram is commutative, $\left(\mathcal{E} \mathcal{M}^{\vee}\right)^{-1} \circ H^{d}(i)(\mathbb{I})=H(\psi)([m])$. By Property 55 , the differential graded algebra $\Omega S_{*}(B G)$ is a Frobenius algebra. More precisely, the morphism $\theta: \Omega S_{*}(B G) \stackrel{\simeq}{\longrightarrow}\left(\Omega S_{*}(B G)\right)^{\vee}, a \mapsto \psi(m) \cdot a$, of right $\Omega S_{*}(B G)$-modules is a quasi-isomorphism.

By Proposition 60, $\phi: \Omega S_{*}(B G) \stackrel{\simeq}{\longrightarrow} B S^{*}(B G)^{\vee}$ is a quasi-isomorphism of 
differential graded algebras. Therefore by i) of Property 57, the square of complexes

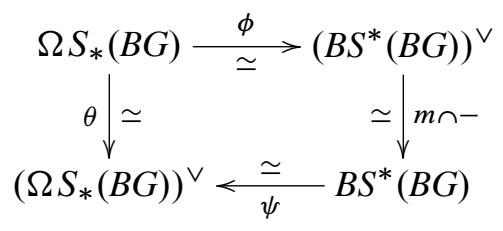

commutes. Therefore (Example 40),

$$
[m] \cap-: \operatorname{Ext}_{S^{*}(B G)}^{p}(\mathbb{k}, \mathbb{k}) \stackrel{\cong}{\Longrightarrow} \operatorname{Tor}_{-d-p}^{S^{*}(B G)}(\mathbb{k}, \mathbb{k})
$$

is an isomorphism.

Let $N$ be any non-negatively upper graded $S^{*}(B G)$-bimodule. Since $B G$ is pathconnected, we obtain from Corollary 14 that the morphism

$$
D^{-1}: \mathrm{HH}^{p}\left(S^{*}(B G), N\right) \stackrel{\cong}{\longrightarrow} \mathrm{HH}_{-d-p}\left(S^{*}(B G), N\right), \quad a \mapsto c \cap a,
$$

is an isomorphism. By taking $N=S^{*}(B G)$ and by passing from a right action to a left action by (30), we obtain a).

The isomorphism $J$ of Jones satisfies $\Delta \circ J=J \circ B$. Since by Lemma 58

$$
B(c)=B \circ J^{-1}(\mathbb{I})=J^{-1} \circ \Delta(\mathbb{I})=0,
$$

we obtain b) from Proposition 32.

\section{Appendix}

The key of the proof of Proposition 32 is the relation

$$
i_{\{a, b\}}=(-1)^{|a|+1}\left[\left[B, i_{a}\right], i_{b}\right]=\left[\left[i_{a}, B\right], i_{b}\right] .
$$

In this appendix we recall that $\left[\left[i_{a}, B\right], i_{b}\right]$ is the derived bracket of $i_{a}$ and $i_{b}$, and we explain that this relation means that the morphism of graded algebras

$$
\mathrm{HH}^{*}(A, A) \rightarrow \operatorname{End}\left(\mathrm{HH}_{*}(A, A)\right), \quad a \mapsto i_{a},
$$

is a morphism of generalized Loday-Gerstenhaber algebras (Theorem 67).

Definition 61 ([24], p. 1247). A generalized Loday-Gerstenhaber algebra is a (not necessarily commutative) graded algebra $A$ equipped with a linear map $\{-,-\}$ : $A_{i} \otimes A_{j} \rightarrow A_{i+j+1}$ of degree 1 such that

a) the bracket $\{-,-\}$ gives $A$ the structure of a graded Leibniz algebra of degree 1 , which means that

$$
\{a,\{b, c\}\}=\{\{a, b\}, c\}+(-1)^{(|a|+1)(|b|+1)}\{b,\{a, c\}\}
$$

for each $a, b$ and $c \in A$, 
b) the product and the Leibniz bracket satisfy the Poisson relation

$$
\{a, b c\}=\{a, b\} c+(-1)^{(|a|+1)|b|} b\{a, c\} .
$$

Proposition 62. Let $A$ be a graded algebra equipped with an operator $d: A_{n} \rightarrow$ $A_{n+1}$ such that $d \circ d=0$ and such that $d$ is a derivation. Then A equipped with the derived bracket (defined by [24], (2.8))

$$
[a, b]_{d}:=(-1)^{|a|+1}[d a, b]
$$

is a generalized Loday-Gerstenhaber algebra.

Proof. Since $A$ is an associative graded algebra, the bracket $[-,-]$ defined by

$$
[a, b]:=a b-(-1)^{|a||b|} b a
$$

is a Lie bracket. Since $d$ is a derivation for the associative product of $A, d$ is a derivation for the Lie bracket [-,-]. Therefore by [24], Proposition 2.1, the derived bracket $[-,-]_{d}$ satisfies the graded Jacobi identity and $d$ is a derivation for the derived bracket $[-,-]_{d}$. Since $[-,-]_{d}$ does not satisfy in general anti-commutativity, $[-,-]_{d}$ is only a Leibniz bracket in the sense of Loday [27] and not a Lie bracket in general. The Lie bracket $[-,-]$ satisfies the Poisson relation

$$
[a, b c]=[a, b] c+(-1)^{(|a|+1)|b|} b[a, c] .
$$

Therefore, since $[a,-]_{d}$ is the derivation $(-1)^{|a|+1}[d a,-]$, the Leibniz bracket $[-,-]_{d}$ also satisfies the Poisson relation ([24], Proposition 2.2)

$$
[a, b c]_{d}=[a, b]_{d} c+(-1)^{(|a|+1)|b|} b[a, c]_{d} .
$$

Remark 63. In Proposition 62, if instead we define the bracket by

$$
[a, b]_{d}:=\operatorname{ad}(b)-(-1)^{(|a|+1)(|b|+1)} b d(a),
$$

then $[-,-]_{d}$ satisfies anti-commutativity and Jacobi: $[-,-]_{d}$ is a Lie bracket $^{1}$ of degree +1 . But this time, $[-,-]_{d}$ does not satisfy the Poisson relation. Note that again $d$ is a derivation for $[-,-]_{d}$.

Proof. Let $a \in A_{x-1}, b \in B_{y-1}$ and $c \in C_{z-1}$ be three elements of $A$ of degrees $x-1, y-1$ and $z-1$. Then

$$
\begin{aligned}
{\left[a,[b, c]_{d}\right]_{d}=} & a d(b d c)-(-1)^{z y} a d(c d b) \\
& -(-1)^{x y+x z} b(d c)(d a)+(-1)^{x y+x z+y z} c(d b)(d a), \\
{\left[[a, b]_{d}, c\right]_{d}=} & a(d b)(d c)-(-1)^{x y} b(d a)(d c) \\
& +(-1)^{z x+z y} c d(a d b)+(-1)^{z x+z y+x y} c d(b d a), \\
(-1)^{x y}\left[b,[a, c]_{d}\right]_{d}= & (-1)^{x y} b d(a d c)-(-1)^{x y+x z} b d(c d a) \\
& -(-1)^{y z} a(d c)(d b)+(-1)^{y z+x z} c(d a)(d b) .
\end{aligned}
$$

\footnotetext{
${ }^{1}$ We could not find this Lie bracket in the literature. So this Lie algebra structure might be new.
} 
Since $d$ is a derivation and $d^{2}=0$, it follows that $d(a d b)=(d a)(d b)$. Hence we have the Jacobi identity:

$$
\left[a,[b, c]_{d}\right]_{d}=\left[[a, b]_{d}, c\right]_{d}+(-1)^{x y}\left[b,[a, c]_{d}\right]_{d} .
$$

Since $[d a, b]_{d}=(d a)(d b)$ and $[a, d b]_{d}=-(-1)^{x(y+1)}(d b)(d a)$, we have

$$
d\left([a, b]_{d}\right)=(d a)(d b)-(-1)^{x y}(d b)(d a)=[d a, b]_{d}+(-1)^{x}[a, d b]_{d} .
$$

This means that $d$ is a derivation for $[-,-]_{d}$.

Example 64 (interior derivation). Let $A$ be an associative graded algebra. Let $\tau \in A_{1}$ such that $\tau^{2}=0$. Then $d:=[\tau,-]$ is a derivation of the associative product and $d \circ d=0$. Therefore, we can apply the previous proposition. In this case, we denote the derived bracket $[a, b]_{d}$ simply by $[a, b]_{\tau}$ and have ([24], Example, p. 1250)

$$
[a, b]_{\tau}=(-1)^{|a|+1}[[\tau, a], b]=[[a, \tau], b] .
$$

Corollary 65 ([24], beginning of Section 2.4). Let $E$ be a graded $\mathbb{k}$-module equipped with an operator $B: E_{n} \rightarrow E_{n+1}$ such that $B \circ B=0$. Then $\operatorname{End}(E)$ equipped with the derived bracket $[a, b]_{B}=[[a, B], b]$ is a generalized Loday-Gerstenhaber algebra.

Proof. Apply Proposition 62 and Example 64 to $\operatorname{End}(E)$ equipped with the composition product.

Theorem 66 (implicit in [24], p. 1269-70, pointed out by Krasilshchik). Let $A$ be a Batalin-Vilkovisky algebra. The morphism of graded algebras induced by left multiplication

$$
\Psi: A \rightarrow \operatorname{End}(A), \quad a \mapsto l_{a},
$$

is an injective morphism of generalized Loday-Gerstenhaber algebras.

Proof. Since $A$ is a graded module equipped with an operator $\Delta: A_{n} \rightarrow A_{n+1}$ such that $\Delta \circ \Delta=0$, by Corollary 65 applied to $A$ and to $B=-\Delta$, End $(A)$ equipped with the derived bracket $[f, g]_{-\Delta}=[[f,-\Delta], g]$ is a generalized Loday-Gerstenhaber algebra. By Proposition 28,

$$
l_{\{a, b\}}=-\left[\left[l_{a}, \Delta\right], l_{b}\right]=\left[\left[l_{a}, B\right], l_{b}\right] .
$$

Therefore $\Psi$ is a morphism of generalized Loday-Gerstenhaber algebra.

Theorem 67. Let $A$ be a differential graded algebra.

1) End $\mathrm{HH}_{*}(A, A)$ equipped with the derived bracket

$$
\left.[a, b]_{B}=[a, B], b\right]
$$


is a generalized Loday-Gerstenhaber algebra.

2) The morphism of graded algebras induced by the action

$$
\Phi: \mathrm{HH}^{*}(A, A) \rightarrow \text { End } \mathrm{HH}_{*}(A, A), \quad a \mapsto i_{a},
$$

is a morphism of a generalized Loday-Gerstenhaber algebra. In particular, its image $\Phi\left(\mathrm{HH}^{*}(A, A)\right)$ is a Gerstenhaber algebra.

Proof. Since the Connes boundary $B: \mathrm{HH}_{*}(A, A) \rightarrow \mathrm{HH}_{*+1}(A, A)$ satisfies $B \circ B=0$, we obtain 1) from Corollary 65 .

Since $i_{a b}=i_{a} \circ i_{b}$ (eq. (31)) and $i_{\{a, b\}}=\left[\left[i_{a}, B\right], i_{b}\right]=\left[i_{a}, i_{b}\right]_{B}$, it follows that $\Psi$ is a morphism of a generalized Gerstenhaber-Loday algebra.

Since $\mathrm{HH}^{*}(A, A)$ is a Gerstenhaber algebra, so is $\Phi\left(\mathrm{HH}^{*}(A, A)\right)$.

Remark 68. If $A$ is a differential graded algebra satisfying the hypotheses of Proposition 32, the morphism $\Phi: \mathrm{HH}^{*}(A, A) \hookrightarrow$ End $\mathrm{HH}_{*}(A, A)$ of Theorem 67 is injective and can be identified with the morphism $\Psi$ of Theorem 66 for the Batalin-Vilkovisky algebra $\mathrm{HH}^{*}(A, A)$.

\section{References}

[1] K. Behrend, G. Ginot, B. Noohi, and P. Xu, String topology for stacks. Preprint 2007. arXiv:0712.3857

[2] R. Berger and R. Taillefer, Poincaré-Birkhoff-Witt deformations of Calabi-Yau algebras. J. Noncommut. Geom. 1 (2007), 241-270. Zbl 1161.16022 MR 2308306

[3] G. E. Bredon, Topology and geometry. Grad. Texts in Math. 139, Springer-Verlag, New York 1997. Zbl 0934.55001 MR 1700700

[4] K. S. Brown, Cohomology of groups. Grad. Texts in Math. 87, Springer-Verlag, New York 1994. Zbl 0584.20036 MR 0672956

[5] D. Burghelea and Z. Fiedorowicz, Cyclic homology and algebraic $K$-theory of spaces-II. Topology 25 (1986), 303-317. Zbl 0639.55003 MR 842427

[6] M. Chas and D. Sullivan, String topology. Preprint 1999. arXiv:math/9911159

[7] D. Chataur and L. Menichi, String topology of classifying spaces. Preprint 2007. arXiv:0801.0174

[8] J. Cuntz, G. Skandalis, and B. Tsygan, Cyclic homology in non-commutative geometry. Encyclopaedia Math. Sci. 121, Springer-Verlag, Berlin 2004. Zbl 1045.46043 MR 2052770

[9] Y. Félix, S. Halperin, and J.-C. Thomas, Adams' cobar equivalence. Trans. Amer. Math. Soc. 329 (1992), 531-549. Zbl 0765.55005 MR 1036001

[10] Y. Félix, S. Halperin, and J.-C. Thomas, Differential graded algebras in topology. In Handbook of algebraic topology, North-Holland, Amsterdam 1995, 829-865. Zbl 0868.55016 MR 1361901 
[11] Y. Félix, S. Halperin, and J.-C. Thomas, Rational homotopy theory. Grad. Texts in Math. 205, Springer-Verlag, New York 2001. Zbl 0961.55002 MR 1802847

[12] Y. Félix, L. Menichi, and J.-C. Thomas, Gerstenhaber duality in Hochschild cohomology. J. Pure Appl. Algebra 199 (2005), 43-59. Zbl 1076.55003 MR 2134291

[13] Y. Felix, J.-C. Thomas, and M. Vigué-Poirrier, The Hochschild cohomology of a closed manifold. Publ. Math. Inst. Hautes Études Sci. 99 (2004), 235-252. Zbl 1060.57019 MR 2075886

[14] P. Feng and B. Tsygan, Hochschild and cyclic homology of quantum groups. Comm. Math. Phys. 140 (1991), 481-521. Zbl 0743.17020 MR 1130695

[15] I. M. Gel'fand, Y. L. Daletskiı̌, and B. L. Tsygan, On a variant of noncommutative differential geometry. Dokl. Akad. Nauk SSSR 308 (1989), 1293-1297; English transl. Soviet Math. Dokl. 40 (1990), 422-426. Zbl 0712.17026 MR 1039918

[16] R. Geoghegan, Topological methods in group theory. Grad. Texts in Math. 243, Springer, New York 2008. Zbl 1141.57001 MR 2365352

[17] E. Getzler, Batalin-Vilkovisky algebras and two-dimensional topological field theories. Comm. Math. Phys. 159 (1994), 265-285. Zbl 0807.17026 MR 1256989

[18] V. Ginzburg, Calabi-Yau algebras. Preprint 2006. arXiv:0612139

[19] T. G. Goodwillie, Cyclic homology, derivations, and the free loopspace. Topology 24 (1985), 187-215. Zbl 0569.16021 MR 793184

[20] S. Halperin, Universal enveloping algebras and loop space homology. J. Pure Appl. Algebra 83 (1992), 237-282. Zbl 0769.57025 MR 1194839

[21] A. Hatcher, Algebraic topology. Cambridge University Press, Cambridge 2002. Zbl 1044.55001 MR 1867354

[22] P. J. Hilton and U. Stammbach, A course in homological algebra. 2nd ed., Grad. Texts in Math. 4, Springer-Verlag, New York 1997. Zbl 0863.18001 MR 1438546

[23] J. D. S. Jones, Cyclic homology and equivariant homology. Invent. Math. 87 (1987), 403-423. Zbl 0644.55005 MR 870737

[24] Y. Kosmann-Schwarzbach, From Poisson algebras to Gerstenhaber algebras. Ann. Inst. Fourier (Grenoble) 46 (1996), 1243-1274. Zbl 0858.17027 MR 1427124

[25] J.-L. Koszul, Crochet de Schouten-Nijenhuis et cohomologie. Astérisque 1985, Numéro Hors Série, 257-271. Zbl 0615.58029 MR 837203

[26] T. Lambre, Dualité de Van den Bergh et structure de Batalin-Vilkoviskiĭ sur les algèbres de Calabi-Yau. J. Noncommut. Geom. 4 (2010), 441-457. Zbl 05777174 MR 2670971

[27] J.-L. Loday, Une version non commutative des algèbres de Lie: les algèbres de Leibniz. Enseign. Math. (2) 39 (1993), 269-293. Zbl 0806.55009 MR 1252069

[28] J.-L. Loday, Cyclic homology. 2nd ed., Grundlehren Math. Wiss. 301, Springer-Verlag, Berlin 1998. Zbl 0885.18007 MR 1600246

[29] D. M. Lu, J. H. Palmieri, Q. S. Wu, and J. J. Zhang, Koszul equivalences in $A_{\infty}$-algebras. New York J. Math. 14 (2008), 325-378. Zbl 1191.16011 MR 2430869

[30] S. Mac Lane, Homology. Grundlehren Math. Wiss. 114, Springer-Verlag, Berlin 1963. Zbl 0133.26502 MR 0156879 
[31] J. McCleary, A user's guide to spectral sequences. 2nd ed., Cambridge Stud. Adv. Math. 58, Cambridge University Press, Cambridge 2001. Zbl 0959.55001 MR 1793722

[32] L. Menichi, The cohomology ring of free loop spaces. Homology Homotopy Appl. 3 (2001), 193-224. Zbl 0974.55005 MR 1854644

[33] L. Menichi, Batalin-Vilkovisky algebra structures on Hochschild cohomology. Bull. Soc. Math. France 137 (2009), 277-295. Zbl 1180.16007 MR 2543477

[34] M. Penkava and A. Schwarz, On some algebraic structures arising in string theory. In Perspectives in mathematical physics, Conf. Proc. Lecture Notes Math. Phys., III, Internat. Press, Cambridge, MA, 1994, 219-227. Zbl 0871.17021 MR 1314668

[35] S. F. Siegel and S. J. Witherspoon, The Hochschild cohomology ring of a group algebra. Proc. London Math. Soc. (3) 79 (1999), 131-157. Zbl 1044.16005 MR 1687539

[36] E. H. Spanier, Algebraic topology. Springer-Verlag, New York 1981. Zbl 0477.55001 MR 0666554

[37] M. E. Sweedler, Hopf algebras. W. A. Benjamin, Inc., New York 1969. Zbl 0194.32901 MR 0252485

[38] D. Tamarkin and B. Tsygan, Noncommutative differential calculus, homotopy BV algebras and formality conjectures. Methods Funct. Anal. Topology 6 (2000), no. 2, 85-100. Zbl 0965.58010 MR 1783778

[39] D. Vaintrob, The string topology BV algebra, Hochschild cohomology and the Goldman bracket on surfaces. Preprint 2007. arXiv:math/0702859

[40] C. A. Weibel, An introduction to homological algebra. Cambridge Stud. Adv. Math. 38, Cambridge University Press, Cambridge 1994. Zbl 0797.18001 MR 1269324

Received July 29, 2009

L. Menichi, UMR 6093 associée au CNRS, Université d'Angers, Faculté des Sciences, 2 Boulevard Lavoisier, 49045 Angers Cedex 01, France

E-mail: luc.menichi@univ-angers.fr 